\title{
Las Sonatas para piano de Beethoven a través de los trabajos de edición de Agnes Zimmermann
}

\author{
Beethoven's Sonatas throughout the Editorial Work of \\ Agnes Zimmermann
}

\begin{abstract}
Virginia Sánchez Rodríguez
Universidad de Castilla-La Mancha

Centro de Investigación y Documentación Musical (CIDoM)-Unidad Asociada al CSIC virginia.sanchez@uclm.es ORCID iD: https://orcid.org/0000-0001-8071-2937
\end{abstract}

\section{RESUMEN}

Las Sonatas para piano son, en su conjunto, una de las obras de Ludwig van Beethoven más interpretadas a lo largo de la historia, tanto en público como en el ámbito escolar, pero también una de las que han disfrutado de un mayor número de ediciones a cargo de pianistas y estudiosos de diferentes países. De acuerdo con esta circunstancia, en este trabajo se presenta la edición realizada por la pianista y compositora británica Agnes Zimmermann (1847-1925), una de las mujeres músicas más célebres de su época que, además, mantuvo un estrecho contacto con el legado beethoveniano a lo largo de su carrera. En concreto, a través de fuentes primarias y secundarias, se propone un acercamiento a la figura de esta destacada fémina y un estudio sobre las particularidades de su edición de las Sonatas para piano de Beethoven, publicada por la editorial Novello, Ewer \& Co. en 1873 y cuya reimpresión, revisada, vio la luz en el año 1880.

Palabras clave: Ludwig van Beethoven, Sonatas para piano, Agnes Zimmermann, mujeres en la música, edición musical. 


\section{ABSTRACT}

The Piano Sonatas are, as a whole, one of the works of Ludwig van Beethoven most performed throughout history, both in public and in the school environment, but also one of those that have enjoyed a high number of editions by pianists and scholars from different countries. In accordance with this circumstance, in this work I present the edition by the British pianist and composer Agnes Zimmermann (1847-1925), one of the most famous women musicians of her time who, in addition, maintained a close contact with the Beethovenian legacy throughout her career. Specifically, through primary and secondary sources, I propose an approach to the figure of this exceptional female and a study on the particularities of her edition work on Beethoven's Sonatas, published by Novello, Ewer \& Co. in 1873 and whose reprint, revised, took place in 1880 .

Key Words: Ludwig van Beethoven, Piano Sonatas, Agnes Zimmermann, women in music, musical edition.

Sánchez Rodríguez, V. (2020). Las Sonatas para piano de Beethoven a través de los trabajos de edición de Agnes Zimmermann. Cuadernos de Investigación Musical, (11, número extraordinario), pp. $45-75$.

\section{INTRODUCCIÓN}

Este año 2020 se conmemora el 250 aniversario del nacimiento de Ludwig van Beethoven (1779-1827), una efeméride que, pese a haber coincidido con la primera pandemia del siglo XXI, está acogiendo la celebración de numerosas acciones académicas, dentro de las limitaciones propias de la situación que vivimos. Esto es posible porque, a pesar de que Beethoven es uno de los más grandes compositores de la historia de la música, de que sus obras se programan a lo largo y ancho del globo terráqueo y de que es uno de los autores más conocidos y reconocidos por parte del gran público, la complejidad y el misterio que rodearon sus circunstancias vitales, así como su rico e inmenso legado, aún permiten, como se ha hecho a lo largo de estos doscientos cincuenta años, el desarrollo de biografías (Jacobs, 1970; Kinderman, 1995; Solomon, 2001) y trabajos desde diferentes perspectivas (Cooper, 1998; Burnham \& Steinberg, 2000; Stanley, 2000).

A este respecto, una persona cuya labor profesional estuvo íntimamente ligada al legado Beethoven y a la difusión de su obra fue Agnes Zimmermann (1847-1925). Si bien es cierto que fue especialmente reconocida como pianista en su época, y que también abordó la composición, esta mujer también destacó por su labor editorial y fue la encargada de desarrollar una edición musical de algunas de las obras más interpretadas del genio de Bonn, sus Sonatas para piano. De acuerdo con esta circunstancia, en este artículo se propone-un estudio sobre la vinculación de Agnes Zimmermann con el legado beethoveniano, profundizando, especialmente, en su labor como editora de las mencionadas obras. 
Aunque a lo largo de las próximas páginas se realiza, a través de fuentes secundarias, una aproximación a la presencia femenina en la música, a la protagonista y al legado pianístico de Beethoven, esta investigación, de perfil cualitativo, se vertebra, principalmente, en torno a las fuentes primarias, formadas por la primera y la segunda edición de las Sonatas para piano del coloso alemán a cargo de Zimmermann, junto a una selección de referencias hemerográficas británicas. A pesar de que se pretende reivindicar el papel de esta figura femenina como una música multidisciplinar, de forma específica, el propósito principal de este trabajo comprende un acercamiento a esta edición y un estudio en torno a las decisiones editoriales de Zimmermann sobre las citadas composiciones.

\section{MUJER Y MÚSICA: UN ACERCAMIENTO CALEIDOSCÓPICO}

En las últimas décadas, los estudios dedicados a la reivindicación de la presencia femenina en la música han tratado de solventar su ausencia historiográfica en torno a dos direcciones. Por un lado, se ha observado un incremento en los estudios dedicados a mujeres músicas. A este respecto, y aunque se han intensificado las investigaciones en torno a Barbara Strozzi (1619-1677), Nannerl Mozart (1751-1829) y otras tantas féminas que contaron con una obra de calidad y/o con cierta visibilidad social en su época, Clara Schumann (18191896) continúa a la cabeza de los últimos y más numerosos trabajos centrados en su vida y obra (Reich, 1985; Vries, 1996; Borchard, 2015; Borchard, 2019, entre otros). Por otro lado, también ha comenzado a ofrecerse una mirada de género hacia el hecho musical en torno a contextos y facetas diversas, desde las bases de la musicología feminista (McClary, 1991; Ramos López, 2003), profundizando en aspectos históricos (Drinker, 1995; Pendle, 2001) e incluso ofreciendo una mirada femenina a la música en contextos propios de la era contemporánea, como las músicas populares o el cine (Koskoff, 1989; Whiteley, 2000; Sánchez Rodríguez, 2013).

En relación con la primera línea expuesta, se puede afirmar que el lugar de la mujer a lo largo de la historia de la música ha dependido, en parte, del círculo sociocultural en el que nacía. Y es que aquellas mujeres de una posición socioeconómica baja no tuvieron la opción de la alfabetización y, por tanto, mucho menos el privilegio de una educación musical. Así, en términos generales, las mujeres que tenían conocimientos musicales solían estar vinculadas a los círculos de poder, como el clero - siendo el caso más paradigmático, probablemente, Hildegard von Bingen (1098-1179) por su labor compositiva y su pensamiento musical-, la nobleza -algunas de las más recordadas son, entre otras, la princesa Leonora d'Este (15151575) o a la vizcondesa francesa Clémence de Grandval (1828-1907), que abordaron la composición musical-, de los círculos intelectuales -especialmente si los progenitores eran, además, músicos, como es el caso de Francesa Caccini (1587-1640), que no solo fue la hija de Giulio Caccini (c. 1550-1618) sino que es reconocida como la primera mujer que escribió una ópera, La liberaz̧ione di Ruggiero dall'isola d'Alcina (1625), o Alma Mahler (1879-1964), hija de la cantante Anna von Bergen (1857-1938) y del pintor Emil Jakob Schindler (1842-1892), que, más allá de su matrimonio con Gustav Mahler (1860-1911) y otros intelectuales del momento, presentó una temprana vinculación hacia la música a pesar de que, a lo largo de 


\section{VIRGINIA SÁNCHEZ RODRÍGUEZ}

su vida, escribió poco- y la corte -un ejemplo lo representa la princesa Ana Amalia de Prusia (1723-1787), hija de Federico Guillermo I de Prusia, que llegó a abordar también la escritura de pequeñas obras, algunas de ellas albergadas en la Staatsbibliothek de Berlín-.

De todas las mujeres vinculadas al hecho musical, las compositoras son aquellas que han suscitado un interés y un espacio académico más notable (Jezic, 1988; Álvarez Cañibano, 2008; Beer, 2016; Halstead, 2017). Sin embargo, el mayor número de mujeres adscritas a la música a lo largo de la historia lo ocupan, especialmente, las intérpretes. Conviene recordar que, en términos generales, durante los siglos XVIII y XIX, la principal vinculación de la mujer a la música se producía en el ámbito doméstico, en torno al concepto de los "entretenimientos de salón” (Rieger, 1986, pp. 175-196), fomentando el canto y la interpretación de ciertos instrumentos en el hogar -especialmente, el piano- como una distracción equivalente a la lectura o a la costura y como una forma de agasajar a invitados y de obtener una buena imagen social, con vistas a lograr un matrimonio ventajoso. Eso se debe a que, salvo casos de virtuosas concretas, hasta finales del siglo XVIII y ya avanzado el siglo XIX no se extendió la profesionalización musical femenina, especialmente originada en familias de músicos: "A number of women, most of whom grew up in families of professional artists or musicians, managed to force successful performing career in the earlyand mid-19 ${ }^{\text {th }}$ century" (Lumsden, 2010, p. 918).

En ese contexto cronológico, las pianistas y las cantantes fueron quienes lograron una mayor admiración y consideración social, siendo las prima donnas aquellas más respetadas y admiradas:

Of all the professional women performers in the nineteenth century, the largest number were singers. Most prominent were those who sang opera, but many great vocalists also appeared in public concerts and church music. The opera singers were the great stars of the period: lionized and adored, they had international reputations and appeared in opera houses throughout Europe and North and South America (Reich, 1991, p. 165).

El otro gran ámbito de dominio femenino osciló en torno al piano, el instrumento rey del siglo XIX, especialmente en el ámbito doméstico de aquellas jóvenes de familias pudientes que podían acceder a clases particulares y permitirse contar con un instrumento de un elevado valor y de tales dimensiones como parte del mobiliario burgués. Aunque las féminas dedicadas profesionalmente al piano no fueron muchas en esta cronología, entre las pianistas más destacadas, y a pesar de que Maria Szymanowska (1789-1831) es reconocida como la primera pianista profesional -cuyo estilo performativo y compositivo pudo influir en Chopin (1810-1849), según Dobrzański (2006, p. 114)-, probablemente Clara Schumann representa, de la forma más completa, el prototipo de virtuosa, a tenor de las referencias historiográficas y hemerográficas en torno a su figura, lo que la catapultó a un gran éxito por parte del público y a un reconocimiento por su técnica, llegando a ser destacada como una de las mejores pianistas de Europa por la crítica. 
A pesar de que la composición y la interpretación profesional son las dos facetas más mencionadas en los últimos trabajos, también hubo féminas adscritas a la impresión. Si bien es cierto que la vinculación a las imprentas tenía una connotación mercantil -pues, especialmente, la mayor parte de impresoras eran viudas que heredaban el negocio del esposo fallecido, a menudo tuteladas por sus hijos mayores-, y de que esta labor no es equiparable a la condición intelectual de la escritura o ejecución, no deja de resultar destacable que algunas mujeres regentaran este tipo de negocios, cuya organización y funcionamiento se regían de un modo casi gremial. Un caso de estos lo representa Susana Muñoz, que en el siglo XVII fue impresora de música en la ciudad de Salamanca, una circunstancia admirable debido a la escasez de imprentas con tipografía musical en España (Iglesias, 2002, p. 463) y de acuerdo con los pocos nombres femeninos conocidos asociados a esta labor, caracterizada por el trabajo manual. Igualmente, también regentaron imprentas en la España moderna mujeres como María Ramírez y María de Solórzano (Mazuela, 2012, p. 391, pp. 435-439).

Por supuesto, otras féminas también destacaron por su labor de mecenazgo. A este respecto, el caso de Beethoven es muy significativo. De hecho, de sus Sonatas para piano, núcleo de este trabajo, once de ellas están dirigidas a algunas de sus mecenas, como la princesa Anna Louise Barbara Odescalchi -a quien se referían, cariñosamente, como Babette y cuyo título nobiliario y apellido de soltera eran condesa von Keglević-, la condesa von Browne, la princesa von Lichtenstein, las condesas Giulia Guicciardi y Theresse von Brunsvik, la baronesa Dorothea Ertmann y la señorita Maximiliane Brentano. A pesar de no contar con dedicatoria en estas sonatas, probablemente una de las mecenas más recordadas sea la condesa Josephine Brunsvik (1779-1821), a quien algunos musicólogos consideran "la amada inmortal" (Massin, 1970; Steblin, 1983; Beahrs, 1988, pp. 64-70; Dahlhaus, 1991; Beahrs, 1993, pp. 183-197) y que no solo comenzó siendo su alumna sino que, a lo largo de los años, se convirtió en un gran apoyo para el compositor.

Igualmente, también hubo mujeres que abordaron la edición musical, una labor que radica en el desempeño de "una serie de decisiones fundamentadas, críticas e informadas; en resumen, en el acto de la interpretación. Editar, además, consiste en la interacción entre la autoría del compositor y la autoría del editor" (Grier, 2008, p. 12). La responsabilidad de esta tarea, que puede comprenderse como un proceso de mediación entre la voluntad del compositor y el público, es evidente e implica la necesidad de que el editor cuente con unos conocimientos musicales profundos sobre el estilo compositivo del autor ${ }^{1}$, desarrollando, además, un minucioso trabajo con las fuentes primarias. Según James Grier, precisamente una buena edición depende del respeto a dichos materiales: "el equilibrio exacto presente en cualquier edición es el producto directo del compromiso crítico del editor con la pieza editada y sus fuentes" (2008, p. 12).

\footnotetext{
${ }^{1}$ Conviene aclarar que, si bien es cierto que el testimonio de James Grier expuesto es el ideal de toda edición musical, especialmente desde una mirada actual, el editor no siempre tuvo conocimientos musicales, tal como demuestra la existencia de ciertas erratas, problemas de notación y otros errores en algunos volúmenes publicados a lo largo de la historia, en los que, en esta ocasión, no vamos a particularizar.
} 


\section{VIRGINIA SÁNCHEZ RODRÍGUEZ}

Quizá el ejemplo más recordado de edición musical femenina dirige nuestras miradas, de nuevo, hacia Clara Schumann, que no solo se convirtió en la editora del legado epistolar de Robert Schumann (1810-1856) sino también de la obra musical completa de su marido para Breitkopf \& Härtel (Schumann, 1879-1893), probablemente persiguiendo, además de unos evidentes beneficios económicos adicionales, la difusión de las obras de éste, que precisamente fue una de las preocupaciones a las que la música trató de hacer frente desde su condición de intérprete al programar y ejecutar sus obras cuando todavía no era un autor reconocido. Ahora bien, otras mujeres músicas también han abordado la edición musical en el pasado, a pesar de no haber recibido demasiada atención por parte del ámbito académico. Un caso paradigmático lo representa Agnes Zimmermann, que, además de haber destacado como pianista, fue la editora, entre otras obras, de las Sonatas para piano de Beethoven a finales del siglo XIX para la editorial inglesa Novello, Ewer \& Co.

\section{AgNES ZiMMERMANN Y SU VINCULACIÓN CON BEETHOVEN}

Nacida el 5 de julio de 1847 en Colonia (Alemania), la protagonista de nuestro trabajo desarrolló su existir en Londres (Reino Unido), donde llegó siendo una niña. Con tan solo nueve años, comenzó a estudiar en la Royal Academy of Music de la ciudad bajo la tutela de Cipriani Potter (1792-1871) y Ernst Pauer (1826-1905), convirtiéndose en la que se consideró más joven estudiante del centro: "the youngest but one who has ever entered its portals" (R.A.M. Club Magazine, 1926, p. 21). Además de piano, Zimmermann también cursó estudios de composición en la mencionada institución con George Macfarren (1813-1887) y Charles Steggall (1826-1905), siendo becada en varias ocasiones durante su adscripción académica como estudiante ${ }^{2}$.

La carrera profesional de Zimmermann como pianista comenzó siendo muy joven, pues su debut se produjo en el año 1863, cuando contaba con tan solo dieciséis años y aún no había finalizado sus estudios: "Her first public appearance as a pianist was at the Crystal Palace Concerts in 1863, and for many years her sound musicianly style ensured for her a prominent position on the concert platform" (The R.A.M. Club Magazine, 1926, p. 21). Solo un año después, en 1864, realizó una pequeña gira a lo largo de varias ciudades de Alemania, destacando especialmente el concierto ofrecido en la Gewandhaus de Leipzig, y desde ese momento comenzó a incrementarse su visibilidad en la actividad cultural de Londres, cosechando siempre buenas críticas en las que destacaban su virtuosismo, su expresividad y su pasión, entre otros rasgos de su pianismo. Véase al respecto un fragmento de la crítica publicada tras la interpretación del Concierto para piano y orquesta Op. $25 n^{0} 1$ de Felix Mendelssohn (1809-1847) ofrecido en el Palacio de Cristal de la capital británica en 1865: "We have never heard it throughout better, if so well, played by female hands. She has facility, accent, expression, and fire, without exaggeration" (The Athenaeum, 1865, p. 528). El hecho de que una joven de tan solo dieciocho años abordada una obra de tal dificultad técnica que es, además, una composición concebida poco más de tres décadas antes por parte de

\footnotetext{
2 "The Academy had but few prizes or scholarships in those days, but Miss Zimmermann distinguished herself by carrying off what was then regarded as the Institution's Blue Ribond: The King's Scholarship, in 1860, being re-elected in 1862" (The R.A.M.Club Magazine, 1926, p. 21).
} 
Mendelssohn-, demuestra su virtuosismo y que estaba al tanto de la música que se hacía en su Alemania natal -seguramente inculcada por su maestro, Cipriani Potter, que no solo era un apasionado del ámbito germánico sino también un conocedor de la obra contemporánea de autores alemanes, tal como mencionaremos más adelante -.

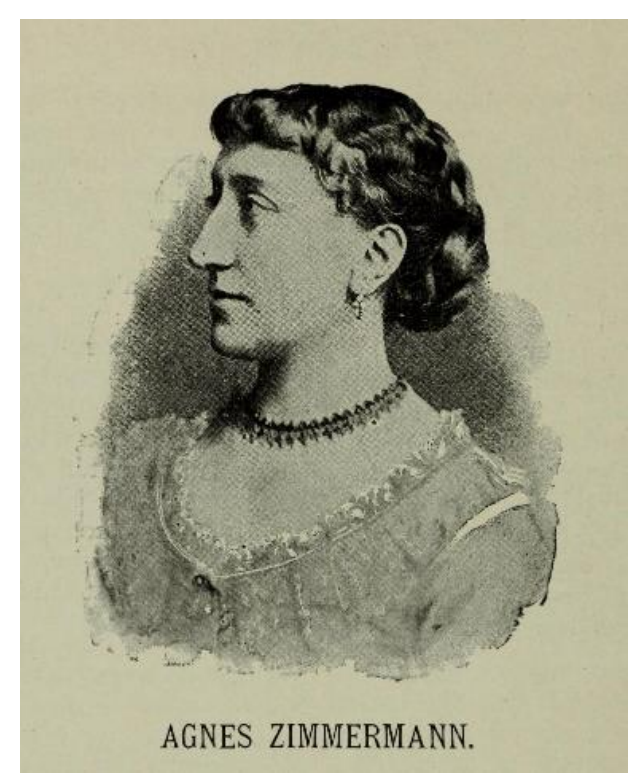

Fig. 1: Agnes Zimmermann (Ehrlich, 1894, p. 366).

De este modo, Zimmermann comenzó a formar parte de la gran oferta cultural del Londres del momento con un repertorio que incluía las grandes sonatas de Beethoven y una selección de composiciones de autores románticos europeos, como Robert Schumann (18101856) o Franz Liszt (1811-1886), entre otros. Su trayectoria como pianista se desarrolló en el elitista círculo musical del momento. De hecho, llegó a compartir programa e interpretaciones con algunos de los virtuosos internacionales, como los violinistas Joseph Joachim (1831-1907) y Wilma Norman-Neruda (1838-1911) o el violonchelista Carlo Alfredo Piatti (1822-1901). También tuvo ocasión de compartir escenario con la propia Clara Schumann, que tan frecuentemente realizó actuaciones en Londres. Ambas tocaron juntas en varias ocasiones desde, al menos, el año $1870^{3}$, siendo especialmente aclamada la interpretación conjunta de las Variaciones sobre un tema de Haydn para dos pianos Op. $56 \mathrm{~b}$ de Johannes Brahms (1833-1897), tal como relata Schumann al propio compositor en una carta con fecha de 5 de mayo de $1876^{4}$ :

\footnotetext{
${ }^{3}$ Clara Schumann, en una carta dirigida a Brahms con fecha de 5 de mayo de 1870, habla de la interpretación junto a Zimmermann de sus Danzas Húngaras en un par de conciertos: "I gave some nice concerts at the end. At two of them we (Frl. Zimmermann and I) played your Hungarian dances, several of which were encored" (Litzmann, 1913, p. 270).

${ }^{4}$ La obra había sido compuesta por Brahms solo tres años antes, en el verano de 1873, por lo que la interpretación de Clara Schumann y Agnes Zimmermann no solo fue absolutamente contemporánea a su escritura de la misma, sino que, seguramente, se trataría de la primera o, al menos, de una de las primeras
} 


\section{VIRGINIA SÁNCHEZ RODRÍGUEZ}

In London I had another Recital: they would not let me off, and it was very successful. It was more crowded than ever, and Chappell cleared $f^{7} 70$ after covering all expenses, including my fee. I played your Variations for two pianos with Miss Zimmermann, and once more revelled in it (Litzmann, 1973, p. 289).

Agnes Zimmermann también formó parte de la programación habitual de los Hanover Square Rooms desde 1865 (Fuller, 2001, p. 835) y, después, de los Saturday Popular Concerts y Monday Popular Concerts de St. James's Hall desde 1875 (Fuller, 1994, p. 345), eventos periódicos celebrados en la desaparecida sala londinense que nutrieron de interpretaciones de calidad a los siempre numerosos asistentes. Probablemente, su habitual presencia en estos actos, junto con la celebración de un concierto anual ${ }^{5}$ en torno a su figura, fueron determinantes para que se convirtiera en una figura reconocida y recordada en la sociedad londinense ya a finales del siglo XIX, descrita como "one of the most justly esteemed of our resident pianists" (The Musical Times and Singing Class Circular, 1891, p. 218), incluso con actividad tras su retirada pública debido a algunos problemas de visión: "After her retirement from concert work, she still kept up her playing in private, though her failing eyesight during the latter part of her life was a serious handicap to her becoming acquainted with new music" (The R.A.M. Club Magazine, 1926, p. 21).

Zimmermann también fue reconocida en su época como compositora, incluso durante su etapa de formación. Su primera obra consistió en la escritura de tres canciones sobre poemas de William Shakespeare (1664-1616), Robert Burns (1759-1796) y Thomas Moore (1779-1852) y de una obertura orquestal en 1863 (Fuller, 1994, p. 345). Precisamente dicha obertura obtuvo una excelente recepción al ser interpretada, posteriormente, en uno de los habituales conciertos de estudiantes gestionados por su escuela, como se observa en una crítica del año 1864:

At the third concert of the Students, which was given at the Hanover Square Rooms, on the $9^{\text {th }}$ ult., the whole of the first part was devoted to the compositions of the pupils. Amongst these, a clever MS. overture, by Miss Agnes Zimmermann, created the greatest effect (...); the silver medals being awarded to Miss Agnes Zimmermann and Mr. Walstein, and the bronze ones to Miss A. Kinkell and Mr. Fox (The Musical Times, 1 de agosto de 1864 , p. 339).

\footnotetext{
interpretaciones de estas Variaciones en la capital británica. A este respecto, las buenas referencias sobre Zimmermann por parte de Schumann habrían sido, seguramente, vitales para el hecho de que Brahms decidiera contar con la joven pianista en la interpretación londinense de esta composición, junto a la ya consagrada Schumann.

${ }^{5}$ Para tomar conciencia de su visibilidad en la época en torno a estas actuaciones anuales, véase un extracto de la crítica del concierto de 1875, donde compartió escenario con lo más granado del momento: “The anual concert of that excellent pianist, Miss Agnes Zimmermann, took place on Thursday evening. The programme included Beethoven's sonata in A, Op. 69, for piano and violoncello, Schumann's "Études Symphoniques", Miss Zimmermann's sonata (No. 2, in A minor) for piano and violin, and Schubert's trio in B flat, the concert giver being assisted by Messrs. Straus and Daubert, and Mdme. Lerumens-Sherrington” (Prout, 1 de mayo de 1875, p. 466).
} 
El estilo académico del que se hizo gala como intérprete desde sus comienzos, y que se mantuvo a lo largo de su vida ${ }^{6}$, también se reflejó en sus composiciones, a pesar de que, en la época, la figura del pianista que, además, era compositor estaba un tanto en declive, como señala Dorothy de Val a tenor de la labor de Zimmermann en comparación con Fanny Davies (1861-1934) y con Dora Bright (1862-1951):

[Fanny] Davies devoted her energy solely to performing, while Bright and Zimmermann were also composers in an era which otherwise saw the decline of the composer-pianist, though the Belgian Juliette de Folville (1870-1946), who became known in England and was a friend of Davies, was an extraordinary combination of pianist, violinist and composer (Val, 2007, pp. 217-218).

En su legado compositivo se observan obras para piano solo -como sus Tres piezas de piano (1865), su Barcarolle Op. 8 (1865-1868) o la Gavotte ${ }^{7}$ en Mi menor Op. 20 (1873)-, obras vocales y también música de cámara -entre la que destacan las Sonatas para violín y piano $O p$. 16, Op. 21 y Op. 23, la Sonata para violonchelo Op. 17 y la Suite para piano, violin y violonchelo Op. 19-, además de transcripciones para piano de obras de Johann Sebastian Bach (1685-1750), George Friedrich Haendel (1685-1759) o Joseph Haydn (1732-1809), autores, estos dos últimos, particularmente vinculados al Londres en el que Zimmermann estaba cosechando tanta fama y prestigio. El hecho de que incluyera su propia música para piano como parte de algunos de los conciertos que ofrecía incidió en la popularización de su legado en una fecha temprana, aunque conviene señalar que, entre todas sus composiciones, de su amplio legado, especialmente fueron laureadas sus obras para violín": "By far the most important name in this field of woman's work [violin composition] is that of Agnes Zimmermann" (Elson, 1903, p. 145).

A pesar de que también ofreció conciertos fuera de Reino Unido, su actividad principal se llevó a cabo en Londres, donde, a lo largo de su vida, compaginó la interpretación musical con la pedagogía en la Royal Academy of Music, la institución en la que ella misma comenzó sus estudios años atrás, como hemos expuesto: "she never discontinued her lessons, and her compositions were often heard at the pupils' concerts at the Academy" (Ehrlich, 1894, p. 367). Quizá precisamente por su dedicación a la pedagogía consideró importante realizar la

\footnotetext{
6 "She occupies a high position in this country as a concert player, and also takes part in the best of classical concerts. From the commencement of her musical career, she was devoted to the classical school of music, and never deviated in her allegiance to it" (Ehrlich, 1894, p. 366).

${ }^{7}$ Ejemplo de la buena acogida del legado de Zimmermann se puede observar en torno a esta Gavotte para piano, que la crítica destacó por su gusto musical y las posibilidades didácticas de estas obras para las estudiantes de la Royal Academy of Music: "Throughout her compositions Miss Zimmermann employs the two hands with much judment" (The Musical Times and Singing Class Circular, 1873, p. 207).

${ }^{8}$ Debemos tener en cuenta que el violín, junto con el piano y la voz, fue uno de los instrumentos con mayor afluencia femenina en Reino Unido a finales del siglo XIX: "Most of the students at England's conservatories in the last decades of the nineteenth century and the beginning of the twentieth were women of whom the great majority studied piano, violin, or voice" (Gillet, 2000, p. 189).
} 


\section{VIRGINIA SÁNCHEZ RODRÍGUEZ}

edición musical de obras, probablemente pensando en ofrecer a los estudiantes textos rigurosos respecto de las fuentes originales pero acordes a los nuevos tiempos.

Tras una vida plenamente dedicada a la música, su fallecimiento se produjo el 14 de noviembre de 1925. Solo unos días después, el 18 de noviembre, se celebró una misa de Requiem en su memoria en St. James's, Spanish Place (The R.A.M. Club Magazine, 1926, p. 21), donde se congregaron numerosos amigos y seguidores. En este punto, conviene subrayar que, a pesar de que su nombre no aparece recogido en la enciclopedia Britannica, de la presencia de escasas líneas en el New Grove y de que, desde su fallecimiento, su figura no ha sido objeto de estudios académicos en profundidad, Zimmermann disfrutó de una gran visibilidad y de un significativo reconocimiento en vida, principalmente por su labor como pianista y compositora, siendo ensalzada también por los grandes virtuosos con los que interactuó en vida. Véase al respecto la opinión del propio Joachim en una fecha temprana, 1868, plasmada en una carta dirigida al músico alemán Theodor Avé-Lallemant (1806-1890):

... Fräulein Agnes Zimmermann is a first-rate pianist, indeed, an artist, and I wish her the success she deserves in Germany with all my heart; she finds it very difficult to make her way in gigantic London. I played the Kreutzer Sonata with her in public in London and was delighted with her performance, but still more with her knowledge of old music and her interest in modern music. She has also composed some very charming things, and a piano and violin sonata dedicated to me will shortly be published, which has, at any rate, many good points. If I were a Concert Director I would speak for her... She is the right kind of musician... (Joachim, 1914, p. 377).

Llegados a este punto, podríamos preguntarnos qué vinculación existe entre Agnes Zimmermann y el legado de Beethoven, de quien celebramos el 250 aniversario de su nacimiento este año. De entrada, conviene señalar que la artista era conocedora de su obra pianística de acuerdo con su faceta como intérprete durante su etapa como alumna y también desde el comienzo de su carrera profesional. De hecho, su debut en el Palacio de Cristal se produjo el 5 de diciembre de 1863 con la interpretación de dos movimientos del Concierto para piano y orquesta en Mi bemol Mayor, «Emperador» (Fuller, 1994, p. 345; Musgrave, 1995, p. 89), obra que interpretó en numerosas ocasiones a lo largo de su carrera ${ }^{9}$. La crítica de esta presentación pública en torno a Beethoven ya destacaba las cualidades de la joven intérprete:

Miss Agnes Zimmermann, a young pianist from the Royal Academy of Music, made a most successful appearance at a concert at the Crystal Palace during the Last month. In the "Andante" and "Rondo" of Beethoven's Concerto in E flat, she fully proved that she had been educated in a good school, and she was warmly and deservedly applauded throughout the performance (The Musical Times and Singing Class, 1 de enero de 1864, p. 205).

\footnotetext{
9 A modo de ejemplo de la interpretación del Concierto «Emperador», véase la reseña de publicada en Musical Notes tras un nuevo concierto celebrado en marzo de 1887 donde, junto a la Sinfonía $n^{\circ}$ 8, «Inacabada», de Schubert, se interpretó la citada obra de Beethoven con Zimmermann al piano (Klein, 1887, p. 32).
} 


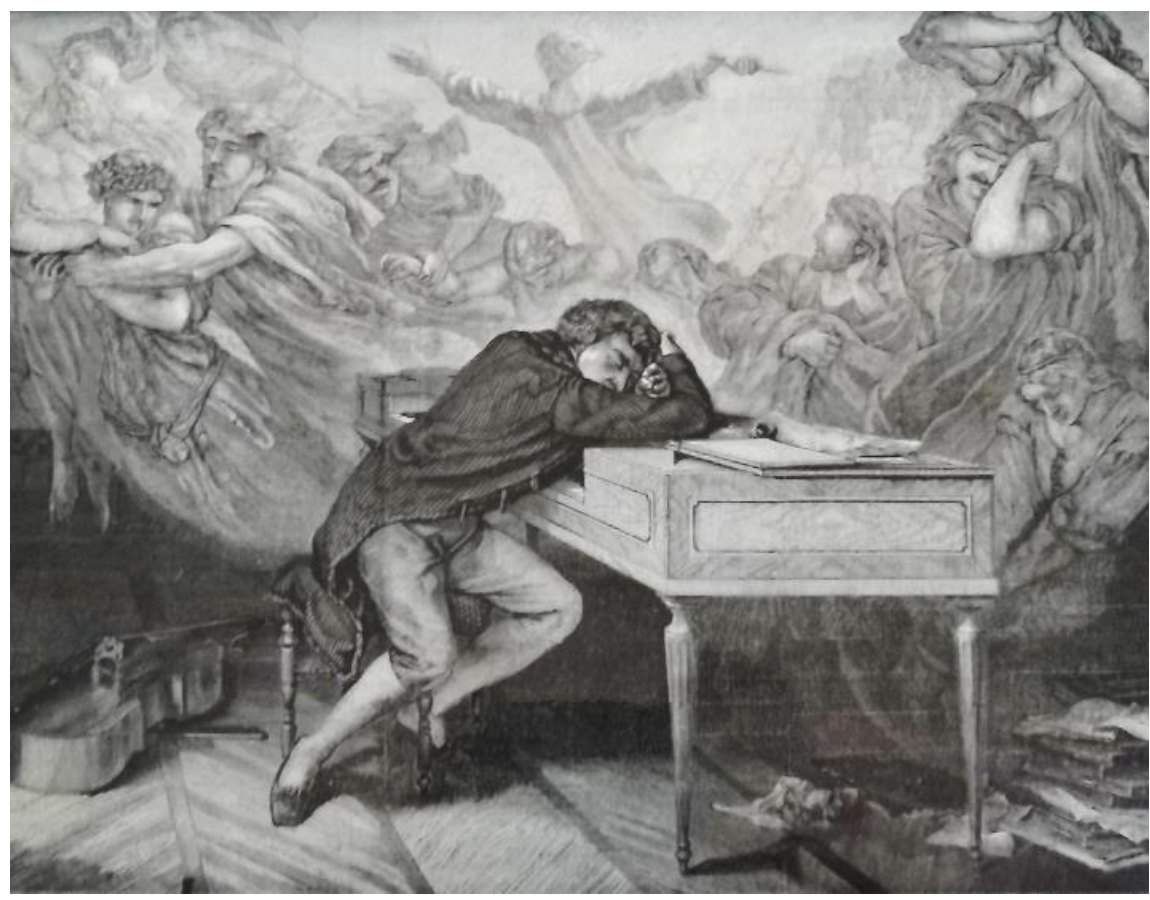

Fig. 2: El sueño de Beethoven, de François-Joseph-Aimé Lemud (1817-1887), grabado.

(Colección Sánchez Rodríguez).

Desde ese instante, la interpretación en público del legado beethoveniano se convirtió en algo habitual de su carrera, una circunstancia que refleja la presencia de sus obras en los programas de concierto en la época -y que es, a su vez, una situación análoga en pleno siglo XXI-. En concreto, Zimmermann no solo fue intérprete de las obras para piano solo sino también de su música de cámara. Respecto de las primeras, las Sonatas para piano ocuparon un lugar destacado, unas obras que aúnan la esencia de la Hausmusik y el virtuosismo, quizá porque "han sido concebidas básicamente como obras privadas o semiprivadas, pero el compositor mismo es un pianista virtuoso con una reputación considerable" (Rosen, 2005, p. 22). Estas obras fueron ejecutadas por Zimmermann en numerosos eventos musicales; entre algunas de las más interpretadas, se encuentran la Sonata Op. $31 n^{\circ} 3$ (Sonata n 18, en Mi bemol Mayor, «La caza», 1802) ${ }^{10}$-caracterizada por esos misteriosos primeros compases que son "los más inestables que Beethoven había escrito hasta entonces" (Rosen, 2005, p. 216)y la Sonata Op. 101 (Sonata $n^{\circ}$ 28, en La Mayor, 1815-1816) ${ }^{11}$-de original estructura, cuyo primer movimiento está formado por una pieza lírica en 6/8-.

Igualmente, Zimmermann también estuvo familiarizada con sus obras para violín y piano, entre otras composiciones camerísticas del coloso de Bonn, siempre acompañada por los más destacados músicos del momento y logrando una gran acogida de público y crítica. A modo ilustrativo, Zimmermann acompañó a Piatti en la Sonata para violonchelo y piano Op. 5

\footnotetext{
${ }^{10}$ Véase al respecto la reseña contenida en el ejemplar de abril de 1887 de Musical Notes (Klein, 1887, p. 38).

${ }^{11}$ Dorothy de Val hace referencia a una interpretación del Op. 101 (Sonata $n^{\circ} 28$ en La Mayor) de Beethoven por parte de Zimmermann el 25 de marzo de 1886, junto a la Fantasía en Do Mayor Op. 17 de Schumann y una selección de composiciones propias (2007, p. 228).
} 


\title{
VIRGINIA SÁNCHEZ RODRÍGUEZ
}

$n^{\circ} 2$ en Sol menor como parte del programa del 10 de enero de 1887 del Monday Popular Concert de St. James's Hall (Klein, 1887, p. 6). Asimismo, como ejemplo de una interpretación camerística junto a otra gran virtuosa de la época, la pianista también acompañó a Wilma Norman-Neruda en la interpretación de la Sonata para violín y piano Op. $21 n^{\circ} 1$ ofrecida en febrero de 1886, también en el marco de los Popular Concerts:

\begin{abstract}
No special interest attached to the chamber concerts given in February. Even the "Pops" presented no feature calling for mention, beyond the introduction for the first time of a Sonata in A minor (Op. 21), for piano and violin, by Miss Agnes Zimmermann -a musicianly work- finely played by the composer and Madame Norman-Neruda, and well received (Klein, 1886, p. 9).
\end{abstract}

En otro orden de cosas, como parte de su dedicación al trabajo sobre composiciones de otros autores, por un lado, destaca la transcripción de obras para piano mencionada anteriormente. Aunque se observa una mayor predilección por autores del Barroco, conviene apuntar que también hizo lo propio con el Concierto para violín y orquesta Op. 61 de Beethoven, cuya aportación ${ }^{12}$ significó, además de la traslación del mensaje musical al registro y las particularidades del piano, la interpretación pública de la obra resultante: "In December 1872, she played a transcription of Beethoven's violin concerto for the first time at a Crystal Palace concert in London" (Ehrlich, 1894, p. 366). Por otro lado, en la década de 1870, la protagonista de este trabajo abordó la elaboración de una nueva edición de sus Sonatas para piano, que es el núcleo de este trabajo.

\section{LaS Ediciones musicales de las Sonatas de BEETHoven a CARgo de AgNeS ZIMMERMANN}

Si bien es cierto que Agnes Zimmermann fue reconocida por su labor performativa y compositiva en Reino Unido, tal como se ha expuesto, también dedicó numerosos esfuerzos a las labores de edición musical. Esta tarea, mencionada por Slonimsky (1978) y Fuller (1994), también fue apreciada por sus coetáneos: "Her fame as a classical pianist was soon established, and her excellent work in editing the sonatas of Beethoven and Mozart bore added testimony to her musical knowledge (...)" (Elson, 1903, p. 145). Como parte de esta adscripción editorial específica, que será comentada más adelante, se puede destacar, junto a las Sonatas de Beethoven que son el núcleo de este trabajo, las Sonatas para piano de Wolfgang Amadeus Mozart (1756-1791) y la obra completa para este instrumento de Robert Schumann, composiciones, todas ellas, sobre las que tenía un profundo conocimiento, en parte debido a su dedicación como pianista.

\footnotetext{
${ }^{12}$ Conviene tener en cuenta que el propio Beethoven ya había realizado una adaptación para piano y orquesta de su obra: el Concierto para piano y orquesta en Re Mayor, Op. 61a, del año 1807 (editado en Viena en 1808 por Comptoir des Arts et de l'Industrie, en tres movimientos y dedicado a Frau [Julie] von Breuning -de soltera, Julie von Vering-. No es posible afirmar si la transcripción de Agnes Zimmermann fue llevada a cabo por desconocimiento de la adaptación pianística del propio compositor o si, aun siendo conocedora de este hecho, Zimmermann decidió realizar una nueva propuesta para piano solo.
} 


\section{DE AgNES ZimMERMANN}

En el año 1873, el panorama musical se enriqueció con la publicación de las Sonatas para piano de Beethoven por parte de la editorial británica Novello, con la que la fémina ya había tenido contacto profesional previamente en relación con la publicación de tres obras vocales propias (The Illustrated London News, 1869, p. 50). El volumen que vio la luz destacó por un cuidado resultado estético y, especialmente, por incluir el mensaje beethoveniano a través de una edición crítica a cargo de Zimmermann. Sobre su función, en términos generales, los editores "estandarizan la notación, adaptan las obras para su interpretación general o para dar respuesta a necesidades institucionales, corrigen errores que les resultan obvios, introducen modificaciones por iniciativa propia” (Grier, 2008, p. 13). Además de estas labores genéricas, Zimmermann, en el volumen protagonista de este artículo, trató de realizar un trabajo contrastado en torno a las mencionadas sonatas, aportando su visión y sus propuestas a partir de su bagaje musical.

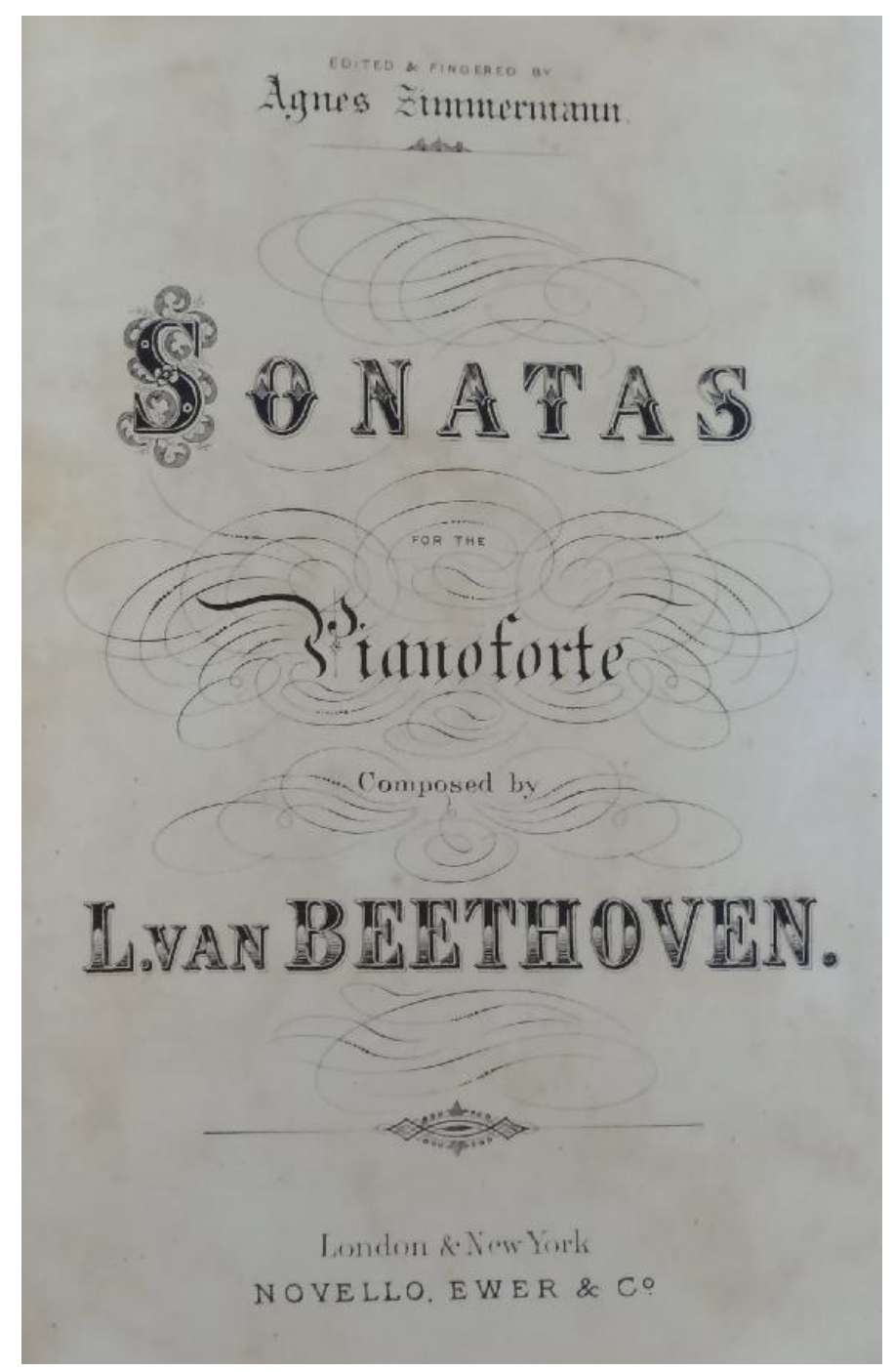

Fig. 3: Sonatas para piano de Beethoven, edición y digitación a cargo de Agnes Zimmermann (1873), portada (Colección Sánchez Rodríguez). 


\title{
VIRGINIA SÁNCHEZ RODRÍGUEZ
}

Agnes Zimmermann abordó la edición de esta titánica obra en un momento propicio en el que se estaba incrementando la popularidad de la obra pianística de Beethoven en Londres, todavía poco normalizada en los planes de estudio de piano. Y es que, a mediados del siglo XIX, las Sonatas para piano de Beethoven aún no gozaban de tanta aclamación en Reino Unido como en la actualidad ${ }^{13}$, algo distinto a la popularidad de sus sinfonías, ya asentadas en la sociedad en las décadas de 1820 y, especialmente, 1830:

\begin{abstract}
While observations of this first decade of the Beethoven symphonies in London are scanty and comparatively incomplete, the stage is set for further development. Hamlet's comment that time is "out of joint" illustrates the theme of this initial decade by serving as a negative comparative. In London, time and conditions seemed to be in joint, and observations reveal that the Beethoven symphonies in London -much unlike Hamlet's aggravated and steady decline- did increase in popularity throughout the second and third decades (Craig, 1985, pp. 76-77).
\end{abstract}

Fueron muchas las instituciones y los profesionales que contribuyeron a la visibilidad del legado del músico de Bonn, siendo, probablemente, la Philarmonic Society of London la más destacada, que incluso llegó a entablar conversaciones con el propio músico para que acudiera al país de Shakespeare, a pesar de que ese viaje nunca llegó a producirse:

\begin{abstract}
And although, as in the music, some of the Philharmonic's most ambitious missioning his works and otherwise sustaining Beethoven be thwarted, the intent as embodied in the votes of and in the decisions of directors was clear. One of the keenest desires of many London musicians was for Beethoven to follow in the foot- steps of Cherubini and (later) Spohr - composing works for the Philharmonic, and appearing in London to direct them. On two occasions the society manifested this dedication to the master by making him offers of three hundred guineas; but, though Beethoven wanted greatly to go, and for long sustained in London a suspenseful anticipation of his coming, the wishes of hosts and guest were never to be fulfilled (Hadley, 1973, p. 450).
\end{abstract}

En lo que respecta a su obra para piano, el descubrimiento y la popularización de sus Sonatas en el Londres de la segunda mitad del siglo XIX se debe, en parte, a la labor de músicos como Cipriani Potter o la propia Agnes Zimmermann. Potter, músico de la Royal Academy of Music y maestro de Zimmermann, conoció a Beethoven y, aunque no logró que el músico alemán se convirtiera en su maestro, sí revisó algunas de sus composiciones e incluso compartió con él consejos y sugerencias. Si bien es cierto que su admiración se puede observar en el artículo titulado "Recollections of Beethoven, with Remarks on His Style",

\footnotetext{
13 Una situación análoga se produjo en la Viena coetánea al compositor: "Durante la época de Beethoven, casi ninguna de sus sonatas para piano se tocaba en público en Viena. La tradición musical vienesa puede haber creado el primer estilo viable de música instrumental pública de la historia de Occidente, pero la ciudad de Viena estaba atrasada en cuanto a la creación de la institución del concierto público -es decir, no los conciertos de bandas o interpretaciones gratuitas al aire libre, sino los conciertos de música instrumental para los que se vendían entradas-" (Rosen, 2005, p. 21).
} 
publicado en 1861 en The Musical Times and Singing Class Circular, Potter, en el texto, trata de desacreditar algunos de los mitos erróneos en torno a la figura del compositor alemán, pero también expone, con satisfacción, que, en los últimos años, se estaba empezando a ofrecer un mayor protagonismo y reconocimiento a su música:

Beethoven's music is now listened to with an attention and delight that his real friends and admirers could scarcely have anticipated. Not unfrequently, indeed, these feelings border on prejudice, since it is impossible that amateurs generally can appreciate those portions of his works, which the cultivated Professor is often at a loss to understand; nevertheless, it is gratifying to witness the anxiety with which the uninitiated endeavour to comprehend what is termed classical writing, emanating from so great a man; exerting their auricular and intellectual faculties, to admire that which, in all probability, is far from being congenial with their predisposed taste and ideas. This prostration of the understanding at the shrine of acknowledged genius, is encouraging to every labourer in the good cause of sterling music, and is the best assurance of a healthy and rational state being at hand (Potter, 1861, p. 150).

Centrándonos en el volumen que nos ocupa, y en relación con las razones que llevaron a Zimmemann a afrontar la edición, ésta expone su voluntad pedagógica en el "Preface" (“Prefacio"):

Notwithstanding the many editions of Beethoven's Sonatas that exist already, the present one will stand in need of no justification if it should prove a help towards the better rendering and clearer understanding of these great works. This it aims to be.

Firstly: by the fingering. Many passages from their complication, present difficulties almost insurmountable to amateurs without some guidance, others again admit of various ways of fingering; in all such cases the one selected is that which the Editress, on careful consideration, believes to be the most conducive to the clearness of the phrasing; and even should it not seem the most easy or simple, the player may be repaid for any practice spent upon it, by a better insight into the purport of such passages.

Secondly: by the slurs, which are of such essential importance to define the phrasing, and yet are so seldom placed with due attention. These have been carefully revised; the best editions have been compared; in innumerable places where (contrary to musical sense and feeling) the slur stops short of the final note or resolution, it is here made to include such note or resolution; in fugal movements where the subject is originally slurred, the same indication is added in all its repetitions.

Thirdly: by the addition of the small staves in some places; it must be borne in mind that the compass of the Pianoforte was formerly much more limited than it is now (...) (Zimmermann, 1880, p. III). 


\section{VIRGINIA SÁNCHEZ RODRÍGUEZ}

Respecto de los tres principales rasgos de la edición presentados por ella misma, en primer lugar, en relación con la digitación, como ella reconoce, va destinada a ayudar a los estudiantes e intérpretes que se aproximan a la obra. Conviene señalar que, en el siglo XIX después de que se sentaran las bases para utilizar los cinco dedos de la mano en la interpretación de instrumentos de tecla-, en Gran Bretaña aún utilizaban el sistema de digitación inglesa ${ }^{14}$, consistente en el empleo de una cruz $(+)$ para hacer referencia al empleo del dedo pulgar y en la numeración de los restantes dedos de la mano, desde el índice hasta el meñique, del 1 al 4 -frente al sistema de digitación continental, basado en la numeración de todos los dedos de la mano, desde el pulgar, con los números del 1 al 5-. Si bien es cierto que la continuación de este sistema en 1873, año de la edición, es un símbolo retardatario, Zimmermann se acogió a la digitación inglesa, seguramente, consciente del potencial comprador nacional del volumen, a pesar de que era conocedora del sistema continental que ella misma empleó en la edición de la obra pianística completa de Schumann años después.

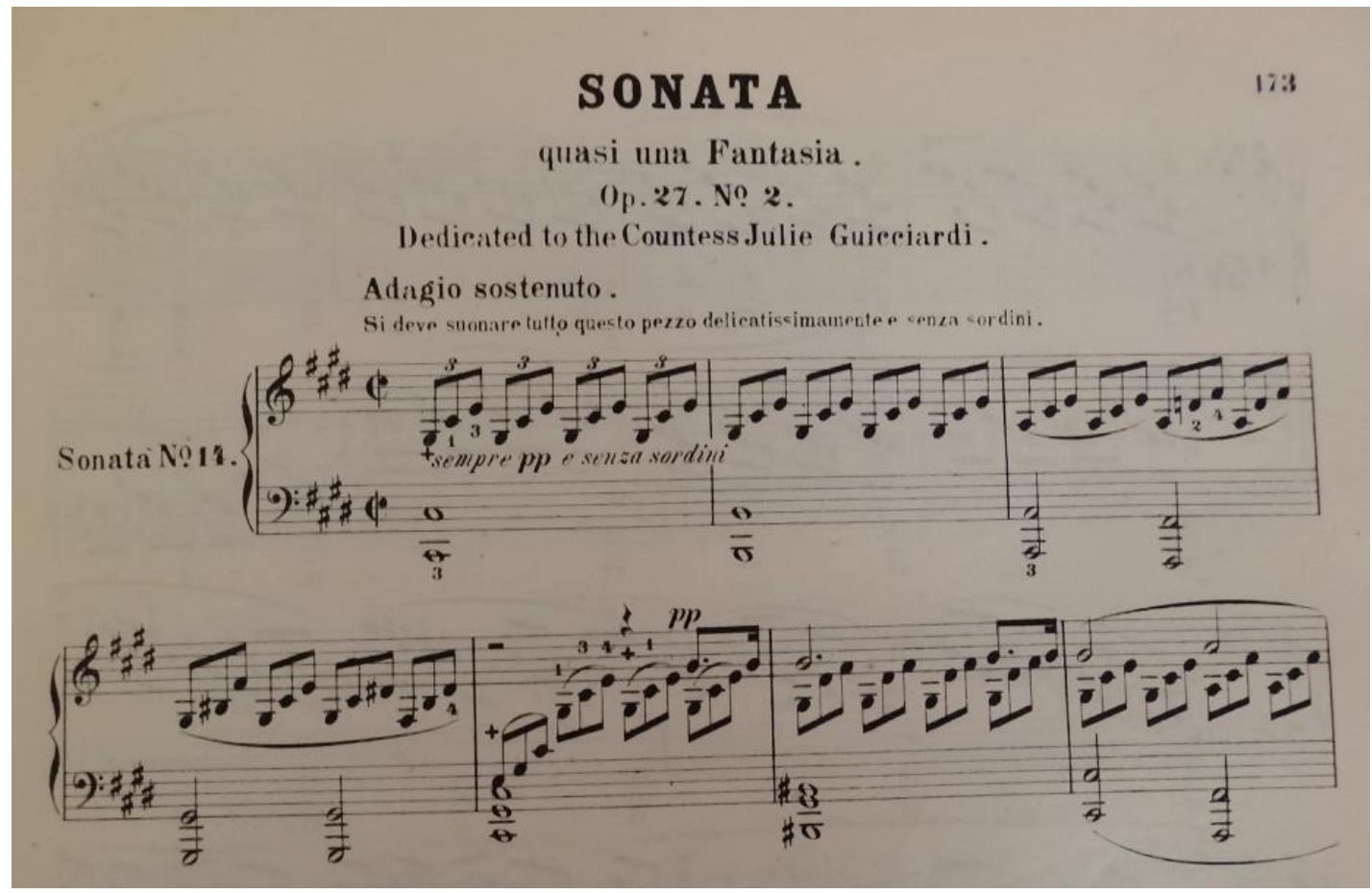

Fig. 4: Sonata Op. $27 n^{\circ} 1$ (Sonata n 14, en Do sostenido menor), "Quasi una Fantasía», edición de 1873 a cargo de Agnes Zimmermann, donde se puede observar la digitación inglesa desde el comienzo, cc. 1-7 (Beethoven, 1873, p. 173).

Sobre las ligaduras de expresión, Zimmermann realiza su propia propuesta tras la revisión de ediciones anteriores. Conviene apuntar que la editora, en el "Preface", no menciona qué fuentes exactas ha tenido en cuenta -pues simplemente expone que ha cotejado y comparado "las mejores ediciones", sin citar si ha tenido acceso a los manuscritos o si ha accedido a la primera edición de todas las Sonatas. De hecho, la editora únicamente

\footnotetext{
14 Para una profundización sobre los diferentes sistemas de digitación, véase Musafia (1971).
} 
hace referencia, de forma específica, a las fuentes cotejadas en la nota crítica de la Sonata $\mathrm{Op}$. $2 n^{\circ} 2$ (Sonata $n^{\circ}$ 2, en La Mayor), señalando que es conocedora de las primeras ediciones y de la última edición de la misma a cargo de Breitkopf und Härtel, así como a la Moscheles y "a otras".

En tercer lugar, como suele ser habitual en las ediciones críticas, la responsable integra algunas versiones alternativas de interpretación. En algunos casos, esas recomendaciones son insertadas en ossia, como pequeñas sugerencias reflejadas en un tamaño de fuente inferior y que son aportaciones encaminadas a una especie de actualización de las Sonatas registro del piano de la segunda mitad del siglo XIX, con mayores posibilidades técnicas que el piano de Beethoven, según el propio "Preface". Tengamos en cuenta que el instrumento, en la época del compositor, tan solo alcanzaba un $\mathrm{Fa}_{5}$ en el índice acústico francobelga, de ahí que Zimmermann, en ocasiones, llegue a hacer otras propuestas de acuerdo con el resto del contenido musical. Véase, a modo de ejemplo, la Sonata Op. $10 n^{\circ} 3$ en el compás 105, donde, en el tercer pulso, Zimmermann propone un $\mathrm{Sol}_{5}$ en lugar de un $\mathrm{Mi}$, imaginando que el compositor lo habría hecho de ese modo en caso de que su piano hubiera contado con el registro oportuno, continuando, de ese modo, el modelo de octava expuesto en el primer pulso de ese mismo compás y en compases previos.

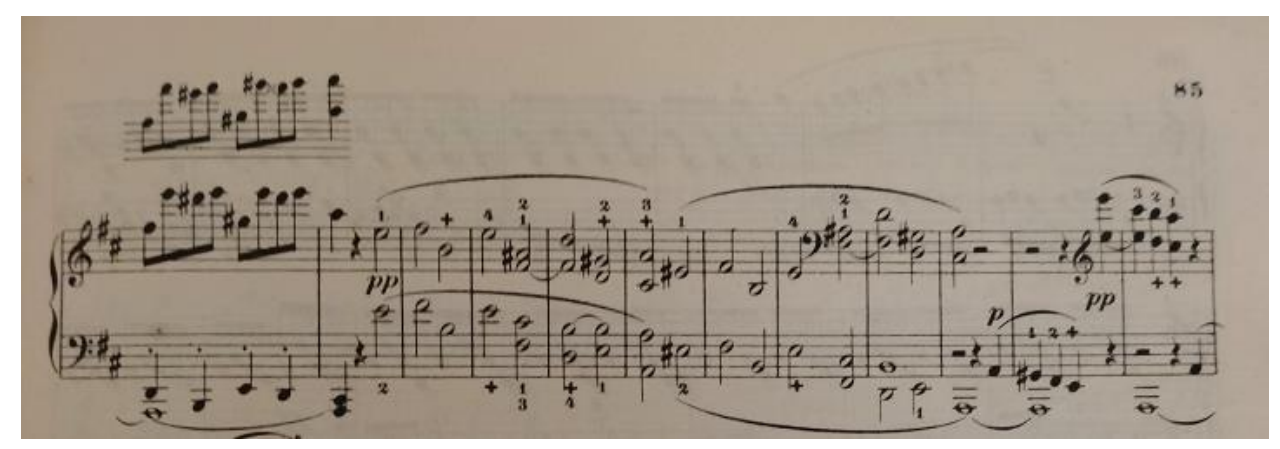

Fig. 5: Sonata para piano Op. $10 n^{\circ} 3$ (Sonata $n^{\circ}$ 7, en Re Mayor), edición de 1873 a cargo de Agnes Zimmermann, cc. 105-116 (Beethoven, 1873, p. 368).

En otros casos, esas modificaciones habrían sido aplicadas directamente, tal como expone la propia Zimmermann en el "Preface":

where it is plain that the mechanical limitations of the instrument alone prevented a complete reproduction of the original passage, such passage is here printed in the shape in which it would probably have been written, had the keyboard in Beethoven's time had its present extent. Such alterations are offered as suggestions only; whoever prefers, can of course play the passage according to the original text. The places where the present edition varies from others in notes, and the reasons for such variations, will be found in the accompanying list (Zimmermann, 1880, p. III). 


\section{VIRGINIA SÁNCHEZ RODRÍGUEZ}

Las notas críticas, expuestas tras el "Preface", especifican aquellas soluciones específicas en torno a la página, la línea y el número de compás, integrando, además, breves ejemplos musicales en ossia cuando corresponde. Esta sistematización es un signo distintivo de la meticulosidad y el respecto de Zimmermann hacia las fuentes originales. Ahora bien, conviene mencionar que la editora no ha incorporado notas de todas las Sonatas, sino únicamente de trece de ellas: Sonata Op. $2 n^{\circ} 1$ (Sonata $n^{\circ}$ 1, en en Fa menor), Sonata Op. $2 n^{\circ} 2$ (Sonata $n^{\circ}$ 2, en La Mayor), Sonata Op. 7 (Sonata $n^{\circ} 4$, en Mi bemol Mayor), Sonata Op. $10 \mathrm{n}^{\mathrm{o}} 1$ (Sonata $n^{\circ}$ 5, en Do menor), Sonata Op. $10 n^{\circ} 2$ (Sonata $n^{\circ}$ 6, en Fa Mayor), Sonata Op. $10 n^{\circ} 3$ (Sonata $n^{\circ}$ 7, en Re Mayor), Sonata Op. 26 (Sonata $n^{0}$ 12, en La bemol Mayor), Sonata Op. 28 (Sonata $n^{\circ}$ 15, en Re Mayor), Sonata Op. $49 n^{\circ} 1$ (Sonata $n^{\circ}$ 19, en Sol menor), Sonata Op. $49 n^{\circ}$ 2 (Sonata $n^{\circ} 20$, en Sol Mayor), Sonata Op. 53 “Waldstein" (Sonata $n^{\circ} 21$, en Do Mayor), Sonata Op. 90 (Sonata $n^{\circ}$ 27, en Mi menor) y Sonata Op. 101 (Sonata $n^{\circ} 28$, en La Mayor) - .

En relación con su criterio, conviene decir que Zimmermann realizó sus decisiones editoriales basándose en un profundo conocimiento del legado de Beethoven, fruto de su labor performativa y de los conocimientos adquiridos durante su carrera también como docente de la Royal Academy of Music. Por ejemplo, la Sonata para piano Op. 106 (Sonata número 29, en Si bemol Mayor), «Hammerklavien», contiene algunos pasajes controvertidos que han dado lugar a diferentes soluciones editoriales, tal como expone James Grier:

Ningún pasaje en la música clásica occidental ha creado más controversia editorial que el puente del primer movimiento de la Sonata «Hammerklavier», Op. 106, de Beethoven (compases 224-226). Los hechos son bien conocidos: dos apuntes contienen la anotación Lał; la primera edición contiene La\#; no se conserva ningún autógrafo. Algunos editores podrán aceptar la anotación que aparece en la copia impresa justificando que ésta debe representar las últimas ideas de Beethoven sobre el tema. Otros podrán alegar, con justificaciones equivalentes, que pueden haber intervenido diversas influencias, distintas a las del pensamiento compositivo del propio Beethoven, para generar la anotación de la copia impresa y, por lo tanto, los apuntes conservan la anotación correcta. De hecho, ambas explicaciones son igualmente posibles. O bien Beethoven cambió de idea entre los apuntes y la primera edición, o bien el becuadro (que anularía al sostenido de la armadura de [la] clave) fue omitido por error en la copia impresa.

Y si el original autógrafo reapareciese, probablemente no decidiría la cuestión. Si concordara con el apunte, Beethoven podría haber cambiado el texto durante el proceso de publicación; si concordara con la copia impresa, podría haberse equivocado mientras escribía el original autógrafo, un caso no exento de paralelos con otras fuentes de la música de Beethoven. Solo si el original [autógrafo] mostrara gráficamente la modificación de Beethoven, quedaría probada la anotación: por ejemplo, si Beethoven hubiese escrito primero un becuadro y luego lo hubiese anulado o borrado, mostrando que comenzó con la idea de especificar un Lał y que, después, cambió conscientemente el texto.

En un caso como éste, los teóricos de la edición normalmente apelarían a las intenciones del compositor. Pero el estado de las fuentes no nos permite conocer las intenciones de Beethoven para tener una certeza sobre la verdad de cuál anotación es la correcta. Tales 
teóricos intentan volver a un momento histórico, el momento en el que Beethoven decidió sobre la anotación del puente, que es simplemente irrecuperable (...).

Ahora los críticos pueden sentirse suficientemente a salvo en esta incertidumbre académica y continuar debatiendo alegremente la valía de las dos lecciones desde esa posición privilegiada, sin comprometerse de una u otra manera. Pero el editor que vaya a publicar esta sonata y, especialmente, el pianista que vaya a tocarla, no disfruta de tal lujo. Para bien o para mal, deben decidir entre La y La\# (...). Este ejemplo muestra que la decisión, no importa cuál sea ni cómo se llega a ella, es la decisión del editor o del intérprete en solitario. No podemos saber cuáles eran las intenciones de Beethoven y lo mejor que podemos hacer es elegir (2008, pp. 11-12).

Conocedora de esta realidad, ante esta situación, Zimmermann optó por mantener el Lah, continuando el criterio de los dos apuntes manuscritos mencionados por Grier -que es, a su vez, la opción más extendidas-también en las ediciones "Urtext" actuales (Beethoven, 1978, p. 518)-, tal como se puede observar en los compases 224 y 225.

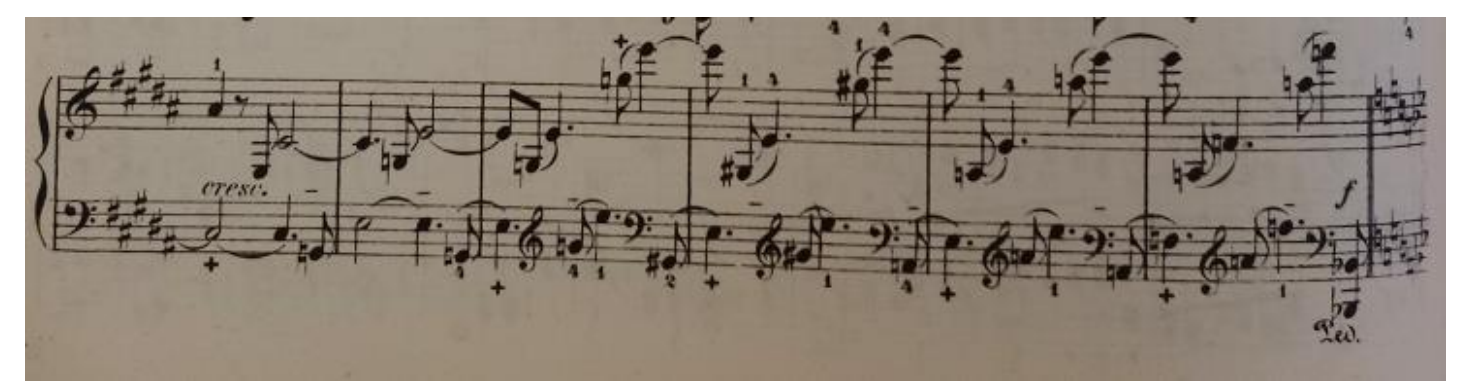

Fig. 6: Sonata para piano Op. 106 (Sonata n²9, en Si bemol Mayor), «Hammerklavien», edición de 1873 a cargo de Agnes Zimmermann, cc. 221-226 (Beethoven, 1873, p. 368).

Junto a las 32 Sonatas para piano, y frente a otras ediciones previas de esta obra completa, Zimmermann también decidió incluir la Sonata [«Leichte Sonate» o Sonatina] para piano en Do Mayor (1830) dedicada a Eleonore von Breuning y las Sonatinas [《Zwei Leichte Sonaten»] en Sol Mayor y en Fa Mayor, de las que se duda su autoría, tal como también menciona la propia Zimmermann. Esta circunstancia determina que el volumen no cuente con el número de sonatas en su título.

La obra, que es, como expone Rosen, "dentro del repertorio de música doméstica, la forma más prestigiosa de música seria” (2005, p. 20), se comercializó en una lujosa edición, con encuadernación granate y detalles dorados. En la cubierta consta el título de la obra, Beethoven's Sonatas, en negro y dorado, recordando el trabajo de los orfebres y plateros realizado en placas y letreros por la grafía empleada y por la propia disposición y decoración de motivos vegetales. Encima y debajo del título, que aparece centrado, se integran dos granadas, símbolo de la editorial, continuando la decoración dorada, que se completa con el remate dorado de las hojas. 


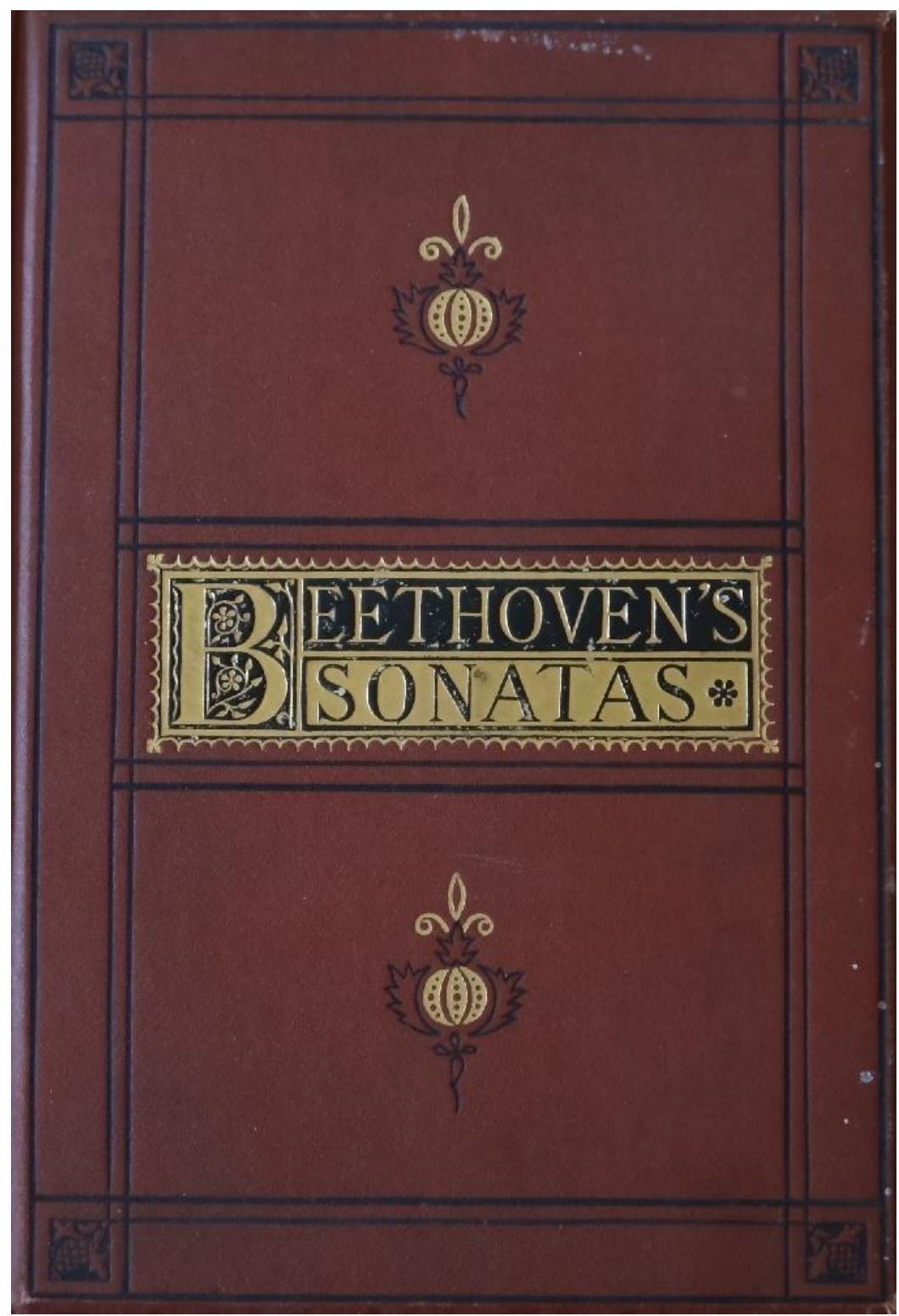

Fig. 7: Sonatas para piano de Beethoven, edición de 1873 a cargo de Agnes Zimmermann, cubierta. (Colección Sánchez Rodríguez).

Unos meses después de su salida al mercado, en enero de 1874, The Musical Times and Singing Class Circular se hizo eco de esta novedad editorial con la publicación de una excelente reseña sobre el volumen. En la crítica, en primer lugar, se destaca el momento propicio para la popularización de las Sonatas para piano de Beethoven, situación que ha sido expuesta anteriormente:

But although the appreciation of these Sonatas is now rapidly increasing, how short a time need we look back to recall the day when in this country they were almost unknown. Many pianists who are now exponents of these works before the public can remember when Monzani and Hill's editions of them were sought for by the few 
students who began to think, or be told by their masters that they were worth attention. "There is no melody in Beethoven", sarcastically remarked Mr. Cipriani Potter, whilst playing some of the most charming portions of the Sonatas to his pupils. "Listen-is this heavy?" he would say, when throwing off, with his fairy-like touch, some of the most beautiful scherzos: and well do we remember his telling one of the students of the Royal Academy of Music to go to the publisher's and ask for the Sonata of Beethoven "that nobody ever played", and his smile of satisfaction when he returned with the right one (The Musical Times and Singing Class Circular, 1 de enero de 1874, p. 363).

En la misma reseña no solo se hacen eco de la lujosa edición y de algunas de las novedades integradas por Agnes Zimmermann desde el punto de vista crítico, sino que, además, ensalzan la figura de la editora de acuerdo con su asentado bagaje musical y su experiencia con el legado beethoveniano:

Miss Agnes Zimmermann, who edits the luxurious edition now before us, has fully earned her right to respect in so responsible a position. An enthusiastic student of Beethoven's works, as well as one of the ablest interpreters of them in public, she has undertaken the duty of editress with a higher feeling than that of merely correcting the proofs, and "seeing the work through the press" (The Musical Times and Singing Class Circular, 1 de enero de 1874, p. 363).

Conjuntamente a las bondades del ejemplar, en esta reseña de 1874 también se indica que las Sonatas pueden adquirirse, además de en su edición completa y lujosa, de forma individual, lo que facilitaba la adquisición de las mismas a los estudiantes, de acuerdo con el elevado coste del volumen. Igualmente, en 1875 también se ofreció a los intérpretes un nuevo formato de adquisición, pues se publicó la edición completa publicada en octavo, manteniendo la esencia y los rasgos originales: "The present issue, in octavo form, is in every respect an exact reproduction of the former one, elegantly bound to correspond with the many similar editions of composers' Works published by the same firm, and of a convenient size to be carried whenever required" (The Musical Times and Singing Class Circular, 1 de noviembre de 1875, p. 268). 


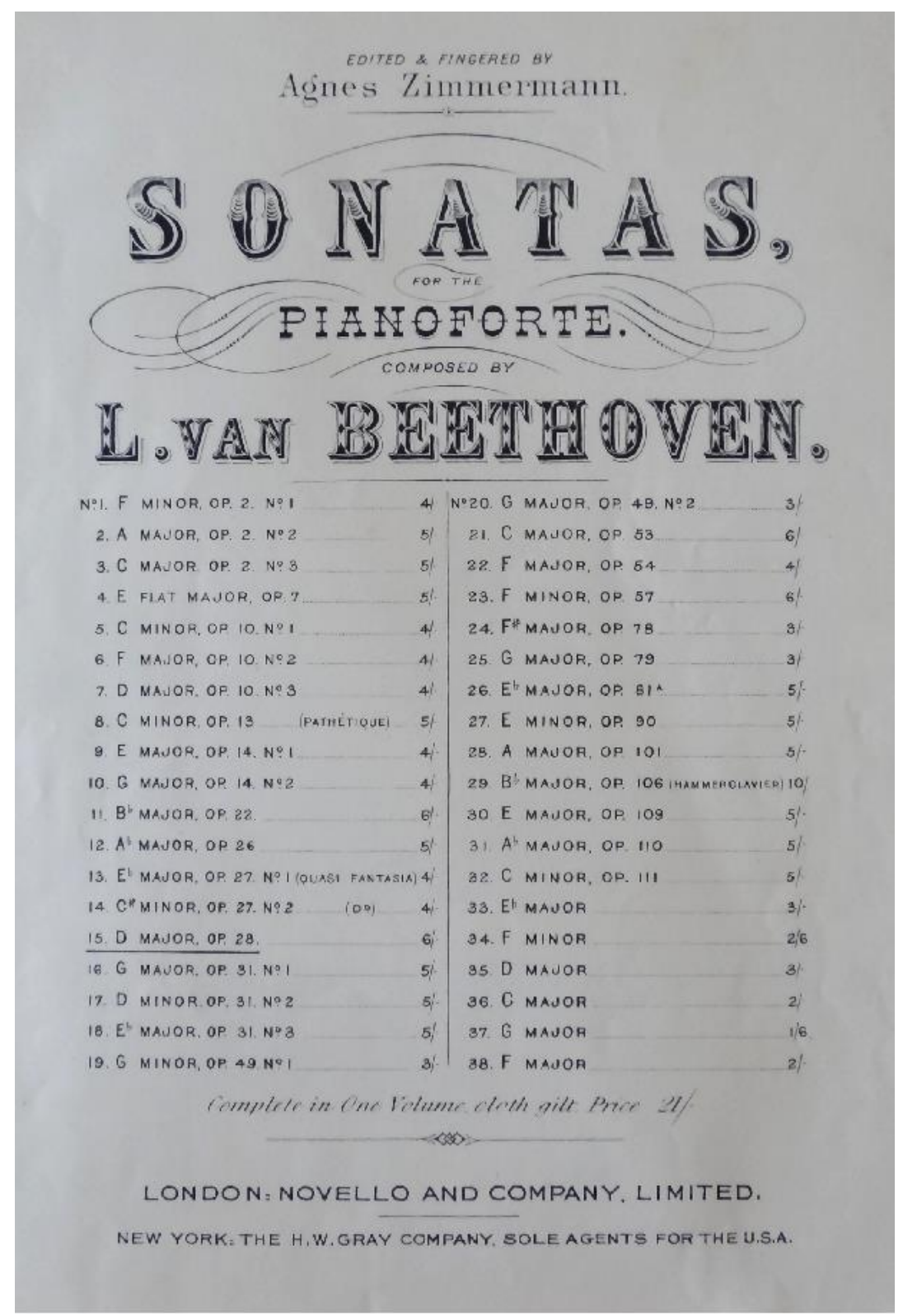

Fig. 8: Sonata para piano Op. 28 (Sonata $n^{\circ} 15$, en Re Mayor), edición y digitación a cargo de Agnes Zimmermann (1873), comercialización individual.

(Colección Sánchez Rodríguez).

El año 1880 fue muy significativo porque es la fecha de publicación de una segunda edición. En lo que respecta a cuestiones estéticas, el volumen era igual, con la diferencia de que su contenido podría ser considerado una versión revisada y mejorada, tal como señala la propia Zimmermann en el "Preface to the Second Edition" fechado en junio de 1880:

In preparing this Edition for reprinting, the Sonatas have again undergone a thorough revision, which has led to some modifications of the first issue. These consist in the lengthening of slurs in many instances, so as to include the whole extent of the phrase; and in various alterations and additions of fingering, all of which, it is hoped, will prove of use both to Students and Teachers (Zimmermann, 1880, p. v). 
Respecto de los rasgos de la primera edición de 1873, la digitación del ejemplar de 1880 continúa el sistema inglés - un hecho que demuestra el convencimiento de Zimmermann sobre las bondades de este sistema de digitación cuya aplicación está centrada, casi exclusivamente, en el ámbito británico- y la única diferencia comprende la inserción de algún añadido o modificación con un sentido pedagógico, para facilitar al intérprete el abordaje de los pasajes respecto de los dedos recomendados. En cuanto a las ligaduras de expresión, en esta edición revisada sufren nuevas modificaciones, concretamente relativas a la prolongación de las mismas, con la intención de mejorar el fraseo y la expresividad. Esto supone una nueva adición respecto de su primer trabajo editorial, pero también respecto de los originales. Véase como ejemplo la Sonata Op. 13 (Sonata $n^{\circ} 8$ ), «Patética». En este caso, y respecto de ediciones anteriores -incluyendo la primera edición, publicada en Austria-, Zimmermann decide ampliar la ligadura hasta la primera nota del compás 5 -en las ediciones anteriores al volumen de Zimmerman, incluyendo la primera edición de 1800 por parte de la editorial Eder, la ligadura finalizaba en el compás 4-, con la intención de completar la extensión de la frase. Asimismo, la editora añade ligaduras de expresión en el compás 5 , con la intención de subrayar los acentos de la figuración semicorchea con puntillo y fusa de la mano derecha, aspectos que tampoco aparecían en ediciones previas.

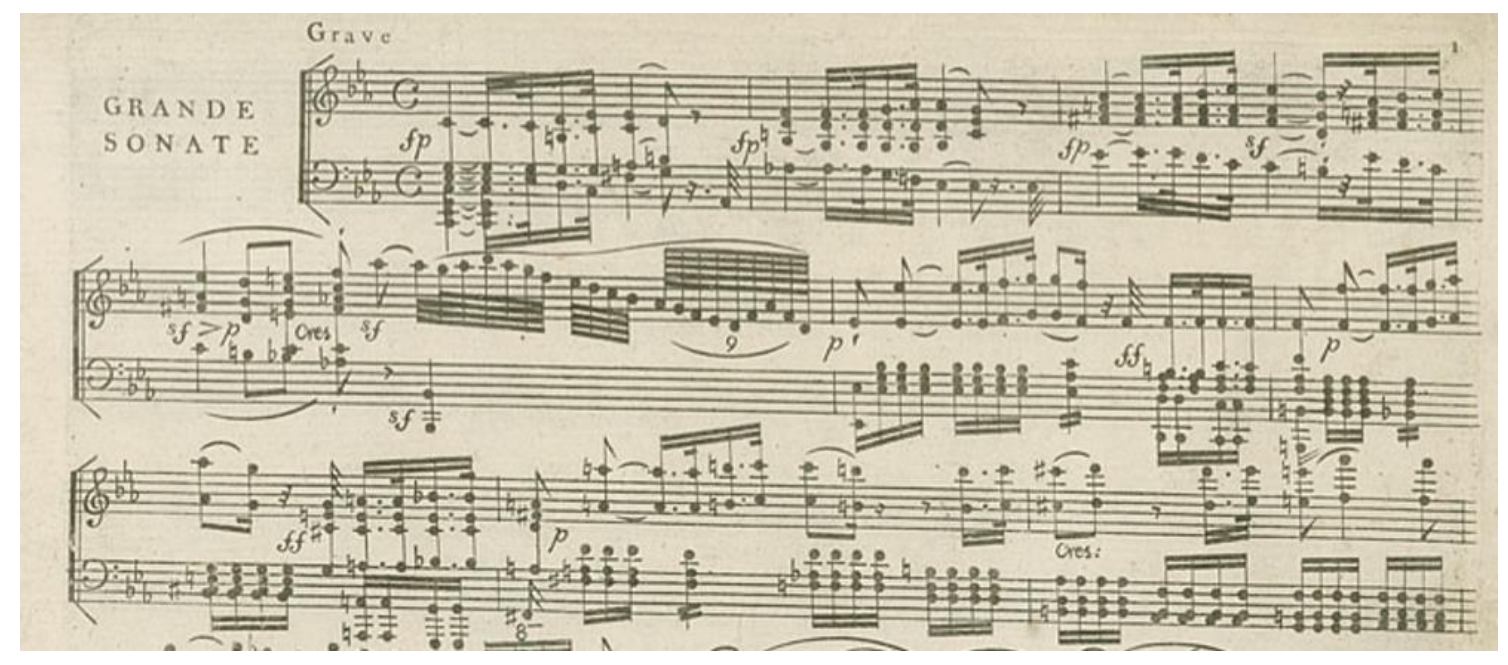

Fig. 9: Sonata Op. 13 (Sonata $n^{\circ}$ 8, en Do menor), «Patética», primera edición a cargo de la editorial austríaca Eder, cc. 1-8 (Beethoven, 1800, p. 1). 


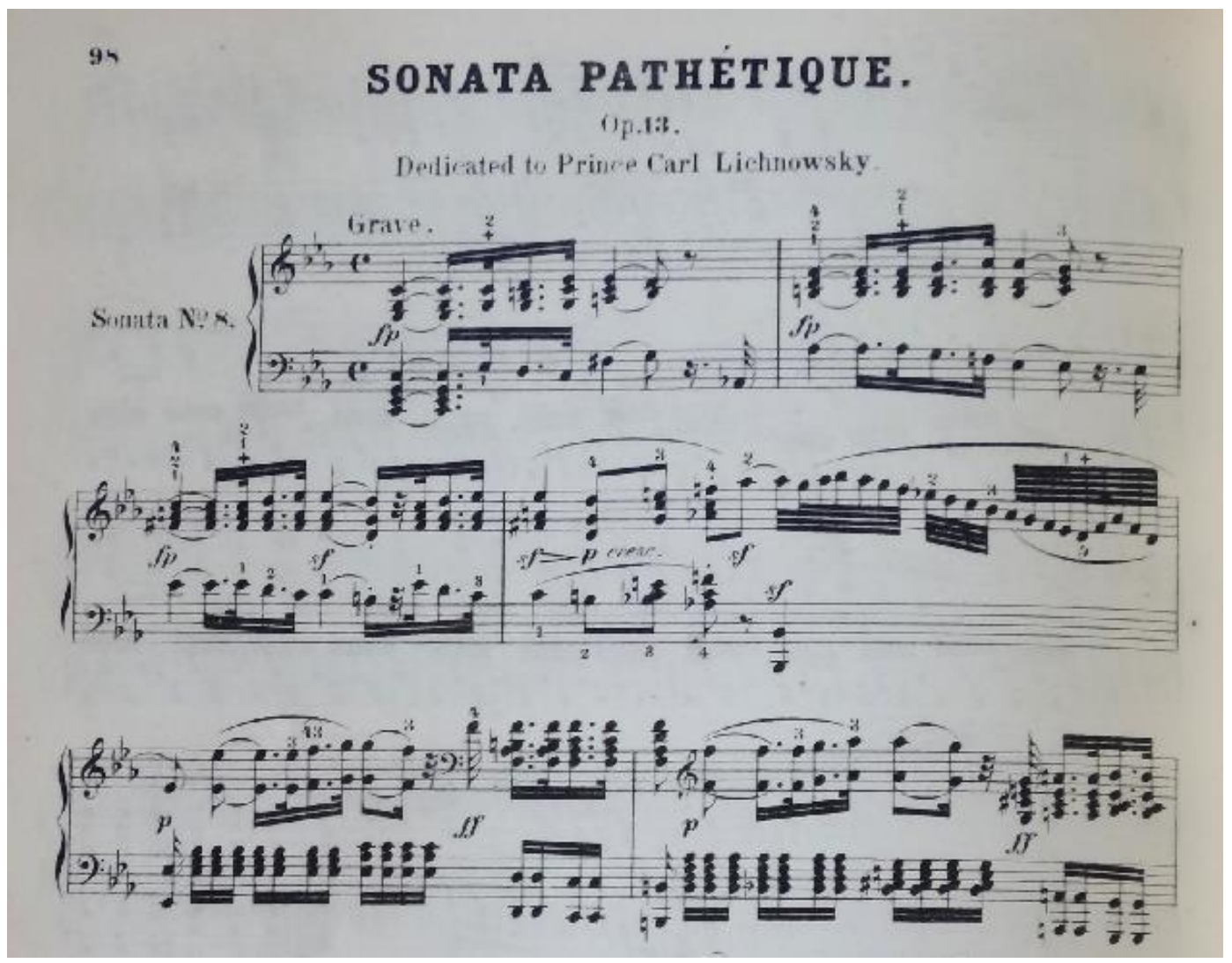

Fig. 10: Sonata Op. 13 (Sonata $n^{\circ} 8$, en Do menor), «Patética», de Beethoven, segunda edición revisada y digitación a cargo de Agnes Zimmermann (1880), cc. 1-6 (Beethoven, 1880, p. 98).

En cuanto a las aportaciones de propuestas interpretativas en ossia, en esta edición de 1880 también se integran. Además, respecto de las notas críticas, Zimmermann incluye algunas modificaciones. En concreto, respecto de la edición de 1873, en esta versión revisada Zimmermann introduce las notas $\mathrm{Fa}$ y Re en el cuarto y sexto pulso del compás 35 de la sexta variación del tercer movimiento de la Sonata Op. 109 (Sonata $n^{\circ}$ 30, en Mi Mayor), sonidos que no fueron recogidos en su edición de 1873 y que sí están en algunas ediciones previas. En ese sentido, se podría considerar que Zimmermann había cambiado de opinión respecto a la solución tomada en la primera edición, una modificación que, sin embargo, no suele recogerse en las ediciones "Urtext" más actuales (Beethoven, 1978, p. 576).

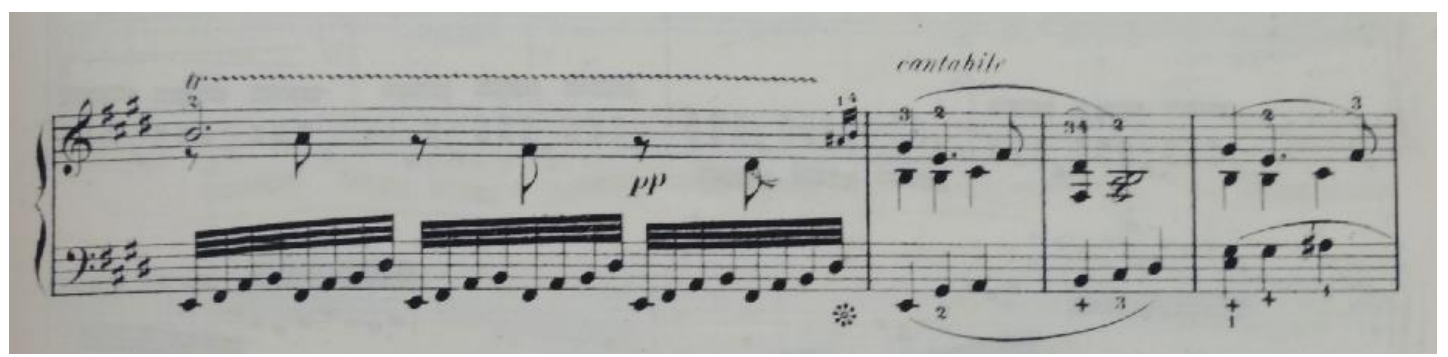

Fig. 11: Sonata para piano Op. 109 (Sonata $n^{\circ}$ 30, en Mi Mayor), segunda edición revisada y digitación a cargo de Agnes Zimmermann (1880), sexta variación del tercer movimiento, c. 35; cc. 1-3 del tema final (Beethoven, 1880, p. 411). 
Conviene decir que, a finales del siglo XIX, Agnes Zimmermann fue también reconocida por esta tarea como editora, algo a lo que, probablemente, también contribuyó su trabajo en torno a la obra que nos ocupa. Así, su nombre era ensalzado como un símbolo de calidad y exactitud, como se puede constatar en la reseña publicada en The Musical Times and Singing Class Circular con motivo de la publicación de la edición de las obras completas para piano de Schumann, lo que demuestra que, tras su trabajo sobre las sonatas de Beethoven y Mozart, Zimmermann era presentada como un argumento de precisión: "Miss Zimmermann's name is itself sufficient to recommend the edition as regards accuracy in the important matter of phrasing marks, \& c., and the suggestion of appropriate fingering" (The Musical Times and Singing Class Circular, 1895, p. 819). Del mismo modo, la edición de Zimmermann sobre las Sonatas para piano de Beethoven fue tenida en cuenta por algunos de los profesionales que realizaron sus propuestas editoriales posteriormente, que no solo conocían sino que incluso referenciaban algunas de sus decisiones, como se puede comprobar en las notas críticas de la Sonata Op. 7 (Sonata $n^{\circ}$ 4, en Mi bemol Mayor) de la edición "Urtext" publicada por Kalmus a finales del siglo Xx (Beethoven, 1996, p. IV). Esta circunstancia demuestra que la edición de Zimmermann ha sido conocido y valorada, al menos en parte, por la comunidad musical.

\section{ConClusiones}

A lo largo de la historia, son muchas las ediciones musicales llevadas a cabo, aunque, como expone Grier, no siempre se han realizado de manera crítica: "La mayor parte de los profesionales interesados en editar hacían simplemente eso, en lugar de preocuparse por detalles metodológicos. Resolvían los problemas ad hoc, producían las mejores ediciones que podían y dejaban de las cuestiones del método a los filólogos” (2008, p. 18). Sin embargo, frente a este testimonio, y superando la existencia de numerosas ediciones no especializadas de décadas y siglos previos, el trabajo realizado por parte de Agnes Zimmermann con este vestigio beethoveniano iba más allá de acuerdo con las decisiones tomadas y aportaciones recogidas.

Con la intención de solventar algunos de los problemas no resueltos en las publicaciones de las Sonatas para piano de Beethoven existentes hasta la fecha, Zimmermann realizó su propuesta de una edición para Novello, Ewer \& Co. en 1873, un contenido que fue, posteriormente, revisado por ella misma en 1880. Además de lo expuesto, el trabajo de edición de Zimmermann puede ser valorado como un ejemplo que demuestra que la edición crítica también ha sido abordada, en ocasiones, por parte de eruditas mujeres músicas, aunque estos trabajos no cuentan, en la mayor parte de los casos, con un lugar en la historiografía. Si bien es cierto que no existen estudios previos sobre Agnes Zimmermann, las escasas y escuetas menciones en torno a esta figura destacada y reconocida en Reino Unido en una cronología coetánea a su existir destacan, especialmente, su labor como pianista y compositora, siempre incidiendo en su buen hacer a colación de lo expuesto en las fuentes hemerográficas, ofreciendo solo una pequeña mención a su labor editorial. 


\section{VIRGINIA SÁNCHEZ RODRÍGUEZ}

Respecto a la edición de las Sonatas para piano de Beethoven que nos ocupa, tras lo expuesto, se puede afirmar que el trabajo de Zimmermann supone una contribución destinada, especialmente, a facilitar a los estudiantes y profesores su interpretación. Eso se observa no solo en las intenciones reflejadas en el "Preface" sino también a través de una cuidada digitación, que es presentada según el sistema inglés, lo que supone un símbolo de arcaísmo. En relación con las decisiones de Zimmermann relativas al contenido musical, la editora propone la «actualización» del contenido musical a las posibilidades del piano de finales del siglo XIX con las sugerencias integradas en ossia y también muestra sus meditadas decisiones ante contradicciones expuestas en ediciones previas, lo que habla de su conocimiento de los volúmenes publicados previamente, desde la primera edición de cada sonata. Conviene aclarar que en este trabajo no se pretende recoger un estudio comparativo entre la edición de Agnes Zimmermann y las ediciones previamente publicadas, sino presentar, a grandes rasgos, las características generales del trabajo editorial de esta mujer, a pesar de que, cuando ha sido necesario, se ha recurrido a ediciones previas para ilustrar, de una forma más precisa, esas decisiones.

Por todo ello, esta edición, inicialmente publicada en 1873 y que contó con una versión revisada en 1880, puede ser considerada un trabajo de interés y éxito que está basado en el conocimiento de Agnes Zimmermann en torno a la obra de Beethoven desde perspectivas diversas, como la interpretación musical profesional, la transcripción y la edición. Así, es posible afirmar, por tanto, que la editora demuestra un profundo dominio de las fuentes primarias con las que trabaja, un respeto hacia el legado de Beethoven y, simultáneamente, un juicio propio basado en sus conocimientos performativos y de teoría de la música. Las decisiones integradas en su edición nos hacen ser conscientes del profundo conocimiento sobre el compositor por parte de Agnes Zimmermann y, a su vez, su compromiso con los futuros intérpretes -al poder ofrecer un texto musical basado en el cotejo de fuentes primarias para la realización de la edición y la posibilidad de aplicar sus conocimientos performativos- y su responsabilidad con el público, puesto que, al fin y al cabo, es el último engranaje del hecho musical.

\section{BibLIOgRAFÍA}

Álvarez Cañibano, A. (Ed.) (2008). Compositoras españolas: la creación musical femenina desde la Edad Media hasta la actualidad. Madrid: Centro de Documentación de Música y Danza.

Beahrs, V. O. (1988). The Inmortal Beloved Riddle Reconsidered. Musical Times, 129(1740), pp. 64-70.

Beahrs, V. O. (1993). Beethoven's Only Beloved? New Perspectives on the Love Story of the Great Composer. Music Review, 54(3-4), pp. 183-197. 
Beer, A. (2016). Sounds and Sweet Airs: The Forgotten Women of Classical Music. London: Oneworld.

Beethoven, L. van (1873). Beethoven's Sonatas [edición y digitación a cargo de A. Zimmermann]. London: Novello, Ewer \& Co.

Beethoven, L. van (1880). Beethoven's Sonatas [edición y digitación a cargo de A. Zimmermann, reimpresión]. London: Novello, Ewer \& Co.

Beethoven, L. van (1978). Beethoven. Sonatas para piano, vol. IV (Wiener Urtext Edition). Madrid: Real Musical.

Beethoven, L. van (1996). Sonatas forpiano nos. 1-7, vol. IA (“Urtext Edition”) [edición a cargo de C. Krebs]. [Miami]: Kalmus.

Beethoven, L. van [1800]. Grande Sonate «Pathétique» pour le clavecín ou PianoForte. Viena: Eder.

Borchard, B. (2015). Clara Schumann. Ibr Leben. Eine biographische Montage. Hildesheim: Olms.

Borchard, B. (2019). Clara Schumann. Musik als Lebensform. Hildesheim: Olms.

Burnham, S. G. \& Steinberg, M. P. (2000). Beethoven and His World. Princeton: Princeton University Press.

Cooper, B. (1998). Beethoven and the Creative Process. Oxford: Clarendon Press.

Craig, K. M. Jr. (1985). The Beethoven Symphonies in London: Initial Decades. College Music Symposium, 25, pp. 73-91.

Dahlhaus, C. (1991). Ludwig van Beethoven: Approaches to his Music. Oxford: Oxford University Press.

Dobrzański, S. (2006). Maria Saymanowska: Pianist and Composer. Los Ángeles: Polish Music Center.

Drinker, S. H. (1995). Music and Women: The Story of Women in Their Relation to Music [1 $1^{\mathrm{a}}$ ed., 1948]. New York: The Feminist Press at The City University of New York.

Ehrlich, A. (1894). Celebrated pianists of the past and present: a collection of one hundred and thirty-nine biographies, with portraits. Manchester: Arthur Livingston.

Elson, A. (1903). Woman's Work in Music: Being an account of her influence on the Art, in Ancient as well as Modern Times. Boston: The Page Company. 


\section{VIRGINIA SÁNCHEZ RODRÍGUEZ}

Fuller, S. (1994). The Pandora Guide to Women Composers: Britain and the United States 1629Present. London \& San Francisco: Pandora.

Fuller, S. (2001). Zimmermann, Agnes (Marie Jacobina). En S. Sadie \& J. Tyrrell (Dirs.). The New Grove Dictionary of Music and Musicians, vol. 27 (p. 835). New York: Grove.

Gillet, P. (2000). Music as a Profession for Women. En P. Gillet (Ed.). Musical Women in England, 1870-1914 (pp. 189-227). New York: St. Martin's Press.

Grier, J. (2008). La edición crítica de la música. Historia, método y práctica [traducción a cargo de A. Giráldez]. Tres Cantos: Akal.

Hadley, D. W. (1973). Beethoven and the Philharmonic Society of London: A Reappraisal. The Musical Quarterly, 59(3), pp. 449-461.

Halstead, J. (2017). The Woman Composer: Creativity and the Gendered Politics of Musical Compositions. London \& New York: Routledge.

Iglesias, A. (2002). El Maestro de Capilla Diego de Bruceña (1567-1623) y el impreso perdido de su Libro de Misas, magnificats y motetes (Salamanca: Susana Muñoz, 1620). En D. Crawford \& G. G. Wagstaff (Eds.). Encomium musicae. Essays in Memory of Robert J. Snow. Hillsdale (pp. 435-470). New York: Pendragon Press.

Jacobs, D. (1970). Beethoven: London: Cassell.

Jezic, D. (1988). Women Composers: The Lost Tradition Found. New York: Feminist Press at the City University of New York.

Joachim, J. (1914). Letters from and to Joseph Joachim. London: MacMillan and Co.

Kinderman, W. (1995). Beethoven. Berkeley: University of California Press.

Klein, H. (1886). Musical Notes: An Annual Critical Record of Important Musical Events. First Issue, Jan.-Dec. 1886. London: Carson \& Comerford.

Klein, H. (1887). Musical Notes: An Annual Critical Record of Important Musical Events. First Issue, Jan.-Dec. 1887. London: Carson \& Comerford.

Koskoff, E. (Ed.) (1989). Music in Cross-Cultural Perspective. Urbana \& Chicago: University of Illinois Press.

Litzmann, B. (1913). Clara Schumann: An Artist's Life Based on Material Found in Diaries and Letters, vol. II. London \& Leipzig: Macmillan \& Company, Breitkopf \& Härtel. 
Lumsden, R. (2010). Women's Leadership in Western Music Since 1800. En K. O'Connor (Ed.). Gender and Women's Leadership. A Reference Handbook, vol. 2 (pp. 917-925). Los Angeles: SAGE Publications.

Massin, J. \& Massin, B. (1970). Ludwig van Beethoven. Madrid: Turner, 1970.

Mazuela Anguita, A. (2012). Artes de canto (1492-1626) y mujeres en la cultura musical del mundo ibérico renacentista. (Tesis Doctoral). Barcelona: Universitat de Barcelona.

McClary, S. (1991). Feminine Endings: Music, Gender, and Sexuality. Minneapolis: University of Minnesota Press.

Musafia, J. (1971). The Art of Fingering in Piano Playing. New York: MCA Music.

Musgrave, M. (1995). The Musical Life of the Crystal Palace. Cambridge: Cambridge University Press.

Pendle, K. (Ed) (2001). Women \& Music: A History. Bloomington \& Indianapolis: Indiana University Press.

Prout, E. (1 de mayo de 1875). Music. Recent concerts. The Academy, p. 466.

Ramos López, P. (2003). Feminismo y Música. Introducción crítica. Madrid: Narcea.

Reich, N. B. (1985). Clara Schumann: The Artist and the Woman. Ithaca \& London: Cornell University Press.

Reich, N. B. (1991). European Composers and Musicians, ca. 1800-1890. En L. Pendle (Ed.). Women \& Music: A History (pp. 147-174). Bloomington: Indiana University Press.

Rieger, E. (1986). ¿Dolce semplice? El papel de las mujeres en la música. En G. Ecker (Ed.). Estética feminista, pp. 175-196. Barcelona: Icaria.

Ringer, A. L. (1970). Beethoven and the London Pianoforte School. The Musical Quarterly, 56(4), pp. 742-758.

Rosen, C. (2005). Las sonatas para piano de Beethoven. Madrid: Alianza.

Royal Academy of Music (1864). The Musical Times, 1 de agosto, p. 390. En The Musical Times and Singing-class Circular, 11. London: Novello.

Sánchez Rodríguez, V. (2013). La banda sonora musical en el cine español y su empleo en la configuración de tipologías de mujer (1960-1969). Salamanca: Universidad de Salamanca. 


\section{VIRGINIA SÁNCHEZ RODRÍGUEZ}

Litzmann, B. (1973). Letters of Clara Schumann and Johannes Brahms 1853-1896, vol. I. New York: Vienna House.

Schumann, R. (1879-1893). Gesamtausgabe der Werke Robert Schumanns (Herausgeberschaft von Clara Schumann). Leipzig: Breitkopf \& Härtel.

Slonimsky, N. (1978). Zimmermann, Agnes. En Baker's Biographical Dictionary of Musicians [6 ${ }^{a}$ ed.], (p. 1947). New York: Schirmer Books.

Solomon, M. (2001). Beethoven. New York: Schirmer.

Stanley, G. (Ed.) (2000). The Cambridge Companion to Beethoven. Cambridge: Cambridge University Press.

Steblin, R. (1983). A History of Key Characteristics in the $18^{\text {th }}$ and Early $19^{\text {th }}$ Centuries. Ann Arbor: UMI Research Press.

The Athenaeum, (1955), 15 de abril de 1865, p. 528.

The Illustrated London News, LV, 1869, p. 50.

The Musical Times and Singing Class Circular, 16(367), 1 de septiembre de 1873, p. 207.

The Musical Times and Singing Class Circular, 16(371), 1 de enero de 1874, pp. 363-364.

The Musical Times and Singing Class Circular, 17(393), 1 de noviembre de 1875, p. 268.

The Musical Times and Singing Class Circular, 36(634), 1 de diciembre de 1895, pp. 818-819.

The Musical Times and Singing Class Circular, 32(577), 1 de abril de 1891, p. 218.

The Musical Times (1864). The Musical Times and Singing Class Circular, vol. XI. London: Novello and Co.

The R. A. M. Club Magazine, 72, marzo de 1926.

Val, D. de (2007). Fanny Davies: 'A messenger for Schumann and Brahms?'. En T. M. Ellsworth \& S. Wollenberg (Eds.). The Piano in Nineteenth-century British Culture: Instruments, Performers and Repertoire (pp. 217-238). Aldershot: Ashgate.

Vries, C. de (1996). Die Pianistin Clara Wieck-Schumann: Interpretation im Spannungsfeld von Tradition und Individualität. Mainz: Schott.

Whiteley, S. (2000). Women and Popular Music: Sexuality, Identity, and Subjectivity. London \& New York: Routledge. 
LAS SONATAS PARA PIANO DE BEETHOVEN A TRAVÉS DE LOS TRABAJOS DE EDICIÓN DE AGNES ZimMERMANN

Zimmermann, A. (1880). Preface. En L. van Beethoven. Beethoven's Sonatas, pp. III-IV. London: Novello, Ewer \& Co.

Zimmermann, A. (1880). Preface to the Second Edition. En L. van Beethoven. Beethoven's Sonatas, p. v. London: Novello, Ewer \& Co.

Fecha de recepción: 11/10/2020

Fecha de aceptación: 13/11/2020 